TRANSACTIONS OF THE

AMERICAN MATHEMATICAL SOCIETY

Volume 353, Number 6, Pages 2215-2243

S 0002-9947(01)02697-6

Article electronically published on February 15, 2001

\title{
THE MONOPOLE EQUATIONS AND $J$-HOLOMORPHIC CURVES ON WEAKLY CONVEX ALMOST KÄHLER 4-MANIFOLDS
}

\author{
YUTAKA KANDA
}

AbStRaCt. We prove that a weakly convex almost Kähler 4-manifold contains a compact, non-constant $J$-holomorphic curve if the corresponding monopole invariant is not zero and if the corresponding line bundle is non-trivial.

\section{Introduction}

The theory of pseudo holomorphic curves has been bringing remarkable progress to both symplectic topology and contact topology since it was initiated by Gromov in $\mathrm{Gr}$.

On the other hand, Witten introduced the monople equations and defined a new invariant of closed orientable smooth 4-manifolds in [W]. Further, he showed that if the 4-manifold $X$ is Kähler, the computation of its invariant can be easily done by using algebraic geometry. The key is the fact that there is a some kind of correspondence between the solutions of the monopole equations on $X$ and the divisors of $X$.

After that, Taubes showed in [T1], T2], T3] that the monopole invariant of a closed symplectic 4-manifold $(X, \omega)$ with $b_{2}^{+}>1$ is equivalent to its Gromov-Witten invariant that counts the "number" of codimension- 1 symplectic submanifolds contained in it.

After that, Kronheimer and Mrowka [K-M2 introduced a suitable analytic setting for the monopole equations on a certain class of non-compact almost Kähler 4-manifolds called A.F.A.K. and extended the definition of monopole invariants to them. Further, as an application, they obtained a striking result on symplectically fillable contact 3-manifolds.

Our main aim is to extend the main result in [T1] to weakly convex almost Kähler manifolds, which are non-compact in general by the definition. Namely, such a manifold contains a compact, non-constant $J$-holomorphic curve if the corresponding monopole invariant is non-zero and if the corresponding line bundle is non-trivial. See Theorem 4.1 in Section 4 for the precise statement. The notion of weak convexity is a slightly stronger condition than that of A.F.A.K. See Definition 1.1 .

Further, in Section 10 we give an application of the main result to contact topology. See Theorem 10.1.

Received by the editors March 8, 1999 and, in revised form, February 28, 2000.

2000 Mathematics Subject Classification. Primary 57R57.

Key words and phrases. Symplectic structure, monopole equation, $J$-holomorphic curve. 
The author would like to thank K.Ono and H.Ohta for several helpful discussions and encouragement.

\section{THE MONOPOLE INVARIANTS OF WEAKLY CONVEX ALMOST KÄHLER 4-MANIFOLDS}

Let $(X, \omega)$ be a symplectic manifold. An almost complex structure $J$ is said to be compatible and the triple $(X, \omega, J)$ is called almost Kähler if the bilinear form $g(*, *):=\omega(*, J *)$ is a $J$-invariant Riemannian metric. It is well known that the space of smooth almost complex structures is contractible under a suitable choice of topology, such as the Whitney topology.

In this paper, we will mainly work on weakly convex almost Kähler 4-manifolds which are defined as follows:

Definition 1.1. An almost Kähler manifold $(X, \omega, J)$ is weakly convex if there exists a proper function $\sigma: X \mapsto[h, \infty)$ with $h>0$ which has the following properties:

Property (A). Any $x \in X$ obeys the conditions below.

1. The injective radius at $x$ is no less than $\sigma(x)$.

2. Let $e_{x}$ be the map $e_{x}: T X_{x} \mapsto X$ defined by $e_{x}(v):=\exp _{x}(\sigma(x) v)$ and let $\gamma_{x}$ be the Riemannian metric on the unit ball in $T X_{x}$ defined by $\gamma_{x}:=$ $\frac{e_{x}^{*}(g)}{\sigma(x)^{2}}$. There exists a sequence of non-negative constants $\left\{c_{k}\right\}_{k \in \mathbb{N}}$ which is independent of $x$ such that the $C^{0}$ norm of the covariant derivatives of order $k$ of $\gamma_{x}$ is bounded by $c_{k}$ for each $k \in \mathbb{N}$.

3. Let $o_{x}$ be the 2 -form on the unit ball defined by $o_{x}:=\frac{e_{x}^{*}(\omega)}{\sigma(x)^{2}}$. There exists a sequence of non-negative constants $\left\{c_{k}^{\prime}\right\}_{k \in \mathbb{Z} \geq 0}$ which is independent of $x$ such that the $C^{0}$ norm of the covariant derivatives of order $k$ of $o_{x}$ is bounded by $c_{k}^{\prime}$ for each $k \in \mathbb{Z}^{\geq 0}$.

4. Let $\hat{\sigma}_{x}$ be the function on the unit ball defined by $\hat{\sigma}_{x}:=\frac{e_{x}^{*}(\sigma)}{\sigma(x)}$. There exists a positive constant $\dot{c}$ which is independent of $x$ such that $\hat{\sigma}_{x} \geq \dot{c}$.

Property (B). There exists a non-negative, integrable function $g_{\sigma}$ of $\mathbb{R}^{\geq 0}$ such that

$$
\int_{\mathbb{R} \geq 0} f g_{\sigma} d y=\int_{X} f \circ \sigma d \operatorname{vol}_{X}
$$

for an arbitrary function $f \in C_{0}^{\infty}\left(\mathbb{R}^{\geq 0}\right)$. Moreover, there exist constants $C>$ $0, \epsilon_{0}>0$ such that $g_{\sigma} \leq C y^{\epsilon_{0}}$. Notice that $g_{\sigma} \equiv 0$ on $[0, h)$.

Remark 1.2. The condition of weak convexity is stronger than that of A.F.A.K. manifolds dealt with in [K-M2]. low:

A typical example of weakly convex almost Kähler 4-manifolds is described be-

Let $X$ be an orientable 4-manifold endowed with conical end, namely, $X$ is diffeomorphic to $X_{0} \cup_{\phi} \partial X_{0} \times[1, \infty)$ where $X_{0}$ is compact with smooth boundary and $\phi$ means the natural identification. Denote $\partial X_{0} \times[1, \infty) \subset X$ by $X^{+}$and $\partial X_{0}$ by $M$. Let $\omega$ be a symplectic form of $X$ which restricts to $X^{+}$as the symplectization of some contact form $\alpha$ of $M$, that is, $\left.\omega\right|_{X^{+}}=d\left(t^{2} \alpha\right)$. Denote by $e_{0}$ the Reeb vector field of $\alpha$. If one chooses a Cauchy-Riemann structure $J^{\prime}$ of the contact plane field $\zeta:=\operatorname{Ker}(\alpha)$ so that $J^{\prime}$ is compatible with $\left.d \alpha\right|_{\zeta}$, this would induce an 
almost complex structure $J$ compatible with $\omega$ over $X^{+}$. In fact, if we identify $T X^{+}$with $T M \oplus T \mathbb{R}^{\geq 1}, J$ is determined by the rules that $J\left(\partial_{t}\right)=e_{0}, J\left(e_{0}\right)=-\partial_{t}$ and that $J(Y)=J^{\prime}(Y)$ if $Y$ is tangent to $\zeta$. Then $J$ extends to the interior of $X_{0}$ so that it is compatible with $\omega$. We can easily check that the pair $(\omega, J)$ satisfies all the conditions of weak convexity. In fact, let $f$ be a smooth Morse function of $X$ such that $\left.f\right|_{X^{+}}=t$ and $f \geq \frac{1}{2}$ on $X$. We may define the function $\sigma$ by $\sigma:=\kappa f$ where $\kappa$ is a sufficently small positive constant.

This example brings us to the following definition.

Definition $1.2([\mathrm{E}])$. A symplectic 4-manifold $\left(X_{0}, \omega\right)$ is a symplectic filling of a contact 3-manifold $(M, \zeta)$ if $\left(X_{0}, \omega\right)$ satisfies the following:

1. $X_{0}$ is compact.

2. $\partial X_{0}=M$ as oriented manifolds.

3. $\left.\omega\right|_{\zeta}$ is non-degenerate.

Recall that in general a contact structure induces a canonical orientation to the base manifold if its dimenson is $4 n+3$. (But in this case, there is no canonical orientation for the contact plane field.)

Proposition 1.3 ([K-M2 $)$. Let $\left(X_{0}, \omega\right)$ be a symplectic filling of a contact manifold $(M, \zeta)$. We can construct a weakly convex almost Kähler 4 -manifold $(X, \tilde{\omega}, J)$ which admits an embedding $\iota:\left(X_{0}, \omega\right) \mapsto(X, \tilde{\omega})$ such that $\zeta$ is invariant under the action of $\iota^{*}(J)$ and such that $X-\iota\left(\right.$ Int $\left.X_{0}\right)$ is diffeomorphic to $\partial X_{0} \times[1, \infty)$. Moreover, this construction is unique in the sense that two such weakly convex almost Kähler structures can be connected by a smooth 1-parameter family. In particular, if $\left.\omega\right|_{M}$ is exact, $(X, \tilde{\omega}, J)$ can be made so that it is as described above as a typical example.

Let $(X, \omega, J)$ be an almost Kähler 4-manifold. $X$ has a $\operatorname{Spin}^{c}$ structure $s_{\omega}$ that is determined canonically by $\omega$. With this understood, define the set $S(X, \omega)$ as follows:

Definition 1.4. $S(X, \omega)$ consists of the isomorphism classes of the pairs $(s, \varrho)$ where $s$ is a $\operatorname{Spin}^{c}$ structure of $X$ being identified with $s_{\omega}$ outside some compact set through the isomorphism $\varrho$.

As we will see later, $S(X, \omega)$ can be identified with the set of isomorphism classes of complex line bundles that have trivializations outside some compact sets. See Section 3 .

If $(X, \omega, J)$ is weakly convex, it is A.F.A.K. by the very definition. Therefore, following K-M2, we can define its monopole invariant. In our terminology, this invariant is a map $S W:\{(X, \omega, J, s, \varrho)\} \mapsto \mathbb{Z}$ obeying the properties below.

Property (1). $S W\left(X, \omega_{0}, J_{0}, s, \varrho_{0}\right)= \pm S W\left(X, \omega_{1}, J_{1}, s, \varrho_{1}\right)$ if there exists a smooth 1-parameter family $\left\{\left(\omega_{t}, J_{t}, \varrho_{t}\right)\right\}_{0 \leq t \leq 1}$ outside some compact set $K$ such that $\varrho_{t}:\left.\left.s\right|_{K} \mapsto s_{\omega_{t}}\right|_{K}$ are isomorphisms and such that $\left(\omega_{t}, J_{t}\right)$ are almost Kähler structures being weakly convex in the following sense: For some compact $K^{\prime}$ with $K \subset \operatorname{Int} K^{\prime}$, there exists a family of proper functions $\sigma_{t}: X \backslash \operatorname{Int} K^{\prime} \mapsto[h, \infty)$ with $h>0$ such that $\left(\omega_{t}, J_{t}, \sigma_{t}\right)$ satisfies Property (A) for any $x \in X \backslash \operatorname{Int} K^{\prime}$ and Property (B) with $X$ replaced by $X \backslash \operatorname{Int} K^{\prime}$.

In a word, the invariant up to sign depends only on the choice of a $\operatorname{Spin}^{c}$ structure and on the "boundary condition".

Property (2). $S W\left(X, \omega, J, s_{\omega}\right.$, id $)=1$. 
Property (3). Suppose that $S W(X, \omega, J, s, \varrho) \neq 0$. Then $\left\langle c_{1}^{2}\left(L_{s}, \tilde{\varrho}\right)-c_{1}\left(L_{s}, \tilde{\varrho}\right) \cup\right.$ $\left.c_{1}(K),[X]\right\rangle=0$. Furthermore, $\left.\left\langle c_{1}\left(L_{s}, \tilde{\varrho}\right)\right) \cup[\omega],[X]\right\rangle \geq 0$ with equality only if $(s, \varrho) \cong\left(s_{\omega}, \mathrm{id}\right)$.

Here $L_{s}$ stands for the corresponding line bundle to $s$ and $\tilde{\varrho}$ is the trivialization of $L_{s}$ induced by $\varrho$ outside a compact set. The first Chern class of $L_{s}$ is regarded as an element of the compactly support cohomology group of $X$ through $\tilde{\varrho}$. Similary, $[X]$ denotes the generator of the fourth homology group of a locally finite singular chain over $\mathbb{Z}$ whose orientation is compatible with $\omega . K$ in (3) denotes the canonical line bundle of $(X, J)$.

Remark 1.5. Proposition 1.3 means that we can well define $S W$ for the pair of contact 3-manifolds and its simplectic filling.

\section{Monopole Equations on Symplectic 4-MANifolds}

We will review some basic facts about monopole equations, especially those on symplectic manifolds.

1. Let $(X, g)$ be a Riemannian 4-manifold. A monopole equation on $(X, g)$ is a non-linear P.D.E. depending on the choice of a $\operatorname{Spin}^{c}$ structure $s$ of $X$. So we will review the definitions of $\mathrm{Spin}^{c}$ structures and Dirac operators first.

a) $\operatorname{Spin}^{c}$ structure $s$ is a $\operatorname{Spin}^{c}(4)=\frac{\operatorname{Spin}(4) \times \mathrm{U}(1)}{ \pm 1}$ lift of the oriented orthonormal frame bundle $\operatorname{Fr}(T X)$. Through the standard representations of $\operatorname{Spin}^{c}(4), s$ associates the positive (resp. negative) spinor bundle $W_{s}^{+}$(resp. $W_{s}^{-}$). $W_{s}^{ \pm}$is a complex, Hermitian, rank-2 vector bundle endowed with the linear map $\rho: T X \mapsto$ $\operatorname{Hom}\left(W_{s}^{+}, W_{s}^{-}\right)$called Clifford multiplication that obeys the relation $\rho(v)^{*} \circ \rho(v)=$ $-g(v)$ id. The signs of $W_{s}^{ \pm}$are canonically determined by the orientation of $X$.

The $\operatorname{Spin}^{c}(4)$ group appears as the structure group of a 4 -tuple $\left(T X, W_{s}^{+}, W_{s}^{-}, \rho\right)$, which is just the central extension by $\mathrm{U}(1)$ of the structure group $\mathrm{SO}(4)$ of $T X$. Thus we can recover the principle bundle $s$ from the 4-tuple according to the standard argument. Therefore, $\operatorname{Spin}^{c}$ structures are in one-to-one correspondence with the isomorphism classes of spinor bundles.

b) A Spinc(4) connection of $s$ is said to be compatible if the associated connection of $\operatorname{Fr}(T X)$ agrees with the Levi-Civita connection. Let $\nabla^{W}$ be a $\mathrm{U}(2) \times \mathrm{U}(2)$ connection on the spinor bundle $W_{s}:=W_{s}^{+} \oplus W_{s}^{-}$. Then $\nabla^{W}$ is a $\operatorname{Spin}^{c}(4)$ connection if and only if the subbundle $\rho(T X) \subset \operatorname{Hom}\left(W_{s}^{+}, W_{s}^{-}\right)$is preserved by the induced connection $\nabla^{\text {Hom }}$ and is compatible if and only if $\left.\nabla^{\mathrm{Hom}}\right|_{\rho(T X)}$ agrees with (the push-forward of) the Levi-Civita connection.

The splitting $\mathfrak{s p i n}^{c}(4)=\mathfrak{s o}(4) \oplus \mathfrak{u}(1)$ implies that a $\operatorname{Spin}^{c}(4)$ connection is determined by choosing a U(1) connection of the determinant line bundle $L_{s}:=$ $\operatorname{det}\left(W_{s}^{+}\right)\left(=\operatorname{det}\left(W_{s}^{-}\right)\right)$. Therefore, the space of a compatible $\operatorname{Spin}^{c}$ connection is an affine space modelled by the space of pure imaginary 1 -forms.

c) With a compatible $\operatorname{Spin}^{c}(4)$ connection $\nabla_{B}$ given, where $B$ stands for the corresponding $\mathrm{U}(1)$ connection of the determinant line bundle, the Dirac operator $\mathcal{D}_{B}$ is defined to be the composition of the sequence

$$
\Gamma\left(W_{s}^{+}\right) \stackrel{\nabla_{B}}{\longrightarrow} \Gamma\left(T X^{*} \otimes W_{s}^{+}\right) \stackrel{\text { Conto }(\rho \otimes i d)}{\longrightarrow} \Gamma\left(W_{s}^{-}\right),
$$

where we identify $T X$ and $T^{*} X$ with each other and Cont stands for the contraction. 
d) The monopole equation for a chosen $\operatorname{Spin}^{c}$ structure $s$ is the one with variables $(\Phi, B) \in \Gamma\left(W_{s}^{+}\right) \times \mathcal{A}\left(L_{s}\right)$ written as follows:

$$
\begin{aligned}
& \mathcal{D}_{B} \Phi=0, \\
& \rho\left(F_{B}^{+}\right)=\left(\Phi \Phi^{*}\right)_{0} .
\end{aligned}
$$

Here $\mathcal{A}\left(L_{\mathfrak{s}}\right)$ denotes the space of $\mathrm{U}(1)$ connections of the determinant line bundle, $F_{B}^{+}$is the self-dual part of the curvature 2 -form of $B$ and $\rho: \Lambda^{2} \mapsto \operatorname{End}\left(W_{\mathfrak{s}}\right)$ is the natural extension of the Clifford multiplication. The subscript ' 0 ' means the traceless part of the said endomorphism.

This equation is equivariant under the action of the gauge group

$$
\mathcal{G}:=\operatorname{Map}(X, \mathrm{U}(1))
$$

which acts on $\Gamma\left(W_{s}^{+}\right)$by the multiplication of a complex number and on $\mathcal{A}\left(L_{s}\right)$ by the pulling-back of connections. We can regard $\mathcal{G}$ as the subgroup of the bundle automorphism of $W_{s}$ that respects the Clifford multiplication.

Notice that (2.2.2) consists of gauge invariant terms. If we add an arbitrary pure imaginary self-dual 2-form to the right-hand side of (2.2.2) to perturb the equation, it remains gauge equivariant.

2. Let $(X, \omega, J)$ be an almost Kähler 4 -manifold. Denote by $g_{J}$ the corresponding Riemannian metric. We will see that the monopole equations (2.2) on the Riemannian manifold $\left(X, g_{J}\right)$ can be written in terms of differential forms and Dolbeaut operators.

a) There is a $\operatorname{Spin}^{c}$ structure $s_{\omega}$ canonically determined by $\omega$. This derives from the fact that the natural projection homomorphism pr: $\operatorname{Spin}^{c}(4) \mapsto \mathrm{SO}(4)$ has a canonical inverse homomorphism over the subgroup $\mathrm{U}(2) \subset \mathrm{SO}(4)$. The spinor bundle and the Clifford multiplcation for $s_{\omega}$ can be explicitly written in terms of differential forms. In fact, define $W_{s_{\omega}}^{+}:=\Lambda^{0,0} \oplus \Lambda^{0,2}$ and $W_{s_{\omega}}^{-}:=\Lambda^{0,1}$. The metrics on them are the ones induced by $g_{J}$. The Clifford multiplication is given for $v \in T X_{x}$ by $\rho(v):=\sqrt{2}\left(\left(v^{0,1} \wedge\right)+\left(v^{0,1} \wedge\right)^{*}\right)$. From the more intrinsical viewpoint, the decomposition $W_{s_{\omega}}^{+}=\Lambda^{0,0} \oplus \Lambda^{0,2}$ is just the eigenspace decomposition of $\rho(\omega) \in \operatorname{End}\left(W^{+}\right)$. The corresponding eigenvalues are $-2 \sqrt{-1}$ and $2 \sqrt{-1}$, respectively. Notice that $\omega$ is a self-dual 2 -form with length $\sqrt{2}$.

b) We will see that there are two natural $\mathrm{Spin}^{c}$ connections for $W_{s}$.

Let $\nabla_{J}^{1}$ be the compatible $\operatorname{Spin}^{c}$ connection that projects to $\Lambda^{0,0}$ as the trivial connection $d . \nabla_{J}^{1}$ preserves the decomposition above if and only if the pair $(\omega, J)$ is Kähler. In fact, if it preserves the decomposition, $\rho(\omega)$ is parallel with respect to the induced connection, which implies that $\nabla^{L \cdot C} \omega \equiv 0$. Then it follows that $\nabla^{L \cdot C} J \equiv 0$, that is, $J$ is integrable.

Let $\nabla_{J}^{2}$ be the $\mathrm{U}(2) \times \mathrm{U}(2)$ connection on $W_{s_{\omega}}$ defined by the composition of the sequence

$$
\bigoplus_{p} \Lambda^{0, p} \stackrel{\nabla^{L . C .}}{\longrightarrow} T^{*} X \otimes\left(\bigoplus_{p} \wedge^{\wedge} T^{*} X \otimes \mathbb{C}\right) \stackrel{i d \otimes p r}{\longrightarrow} T^{*} X \otimes\left(\bigoplus_{p} \Lambda^{0, p}\right)
$$

We can check after a short calculation that $\nabla_{J}^{2}$ is indeed a $\operatorname{Spin}^{c}(4)$ connection. This preserves the decomposition $W_{s_{\omega}}^{+}=\Lambda^{0,0} \oplus \Lambda^{0,2}$ and restricts it to $\Lambda^{0,0}$ as the trivial connection $d$, but it is not compatible unless $(\omega, J)$ is Kähler as we have seen before.

c) Define the operators $\mathcal{D}^{1}$ and $\mathcal{D}^{2}$ to be the compositions of the sequence (2.1) with $\nabla^{B}$ being replaced by $\nabla_{J}^{1}$ and $\nabla_{J}^{2}$, respectively. The former is one of the usual 
Dirac operators for $\operatorname{Spin}^{c}$ structures and the latter is written in the following form

$$
\mathcal{D}^{2}=\sqrt{2}\left(\bar{\partial}+\bar{\partial}^{*}\right),
$$

where $\bar{\partial}$ is the Dolbeaut operator. Then we can easily check after a short calculation that $\mathcal{D}^{1}=\mathcal{D}^{2}$ if and only if $d \omega=0$.

d) Fix a $\operatorname{Spin}^{c}$ structure $s$. Then its spinor bundle is given as the tensor product over $\mathbb{C}$ of $W_{s_{\omega}}$ with a suitable complex line bundle $L$. The Dirac operator for $s$ is given by choosing a $\mathrm{U}(1)$ connection $a$ for $L_{s}$ and is written as

$$
\sqrt{2}\left(\bar{\partial}_{a}+\bar{\partial}_{a}^{*}\right)
$$

where $\bar{\partial}_{a}$ means the usual coupled Dolbeaut operator. Note that

$$
\begin{aligned}
& \Lambda^{+} \otimes \mathbb{C}=\Lambda^{2,0} \oplus \Lambda^{0,2} \oplus \mathbb{C}\langle\omega\rangle, \\
& \Lambda^{-} \otimes \mathbb{C}=\Lambda^{1,1} \cap(\mathbb{C}\langle\omega\rangle)^{\perp} .
\end{aligned}
$$

With this understood, the monopole equation corresponding to $s$ is written as

$$
\begin{aligned}
& \bar{\partial}_{a} \alpha+\bar{\partial}_{a}^{*} \beta=0, \\
& 2 F_{a}^{0,2}+F_{\bar{K}}^{0,2}=\frac{1}{2} \alpha^{*} \beta, \\
& \Lambda\left(2 F_{a}+F_{\bar{K}}\right)=\frac{\sqrt{-1}}{2}\left(|\alpha|^{2}-|\beta|^{2}\right),
\end{aligned}
$$

where

$$
(\alpha, \beta, a) \in \Gamma\left(\Lambda^{0,0} \otimes L\right) \times \Gamma\left(\Lambda^{0,2} \otimes L\right) \times \mathcal{A}(L) .
$$

Here $F_{\bar{K}}$ is the curvature of the connection $\left.\nabla_{J}^{2}\right|_{\Lambda^{0,2}}$ and $\Lambda: \Lambda^{p, q} \mapsto \Lambda^{p-1, q-1}$ denotes the adjoint of $\omega \wedge: \Lambda^{p-1, q-1} \mapsto \Lambda^{p, q}$. Notice that $\rho\left(\gamma_{2}\right) \gamma_{1}=2 \gamma_{1} \gamma_{2}$ for $\gamma_{1} \in \Lambda^{0,0}$ and $\gamma_{2} \in \Lambda^{0,2}$.

\section{THE MOdULI SPACES OF MONOPOLE EQUATIONS ON WEAKLY CONVEX ALMOST KÄHLER MANIFOLDS}

Let $(X, \omega, J)$ be a weakly convex almost Kähler 4-manifold.

Our main object is the following equation, which was introduced for the first time by Taubes:

$$
\begin{aligned}
& \bar{\partial}_{a} \alpha+\bar{\partial}_{a}^{*} \beta=0, \\
& F_{a}^{0,2}=\frac{r}{4} \alpha^{*} \beta+\eta^{0,2}, \\
& \Lambda F_{a}=\frac{\sqrt{-1} r}{4}\left(-1+|\alpha|^{2}-|\beta|^{2}\right)+\Lambda \eta .
\end{aligned}
$$

Here $\eta$ is a pure imaginary self-dual 2-form introduced for the equation to be transverse. $r$ is a positive constant which we will call the rescaling parameter.

Remark 3.0. The equation above is obtained from (2.7) by dropping the terms derived from the curvature of the anti-canonical line bundle, adding $-\frac{\sqrt{-1} r}{2}$ to the right-hand side of $(2.7 .3)$ and rescaling $(\alpha, \beta)$ by the factor $\sqrt{r}$. From the more 
intrinsical viewpoint, it is equivalent to the following equation:

$$
\begin{aligned}
\mathcal{D}_{a} \Phi & =0, \\
2 \rho\left(F_{a}^{+}\right) & =r\left\{\left(\Phi \Phi^{*}\right)_{0}-\frac{\sqrt{-1}}{2} \rho(\omega)\right\} .
\end{aligned}
$$

Fix an element $(s, \varrho) \in S(X, \omega)$ and suppose that $W_{s}=W_{s_{\omega}} \otimes L$ for a complex line bundle $L . \varrho:\left.\left.W_{s}\right|_{X \backslash K} \stackrel{\cong}{\cong} W_{s_{\omega}}\right|_{X \backslash K}$ induces $\tilde{\varrho}:\left.L\right|_{X \backslash K} \stackrel{\cong}{\risingdotseq}(X \backslash K) \times \mathbb{C}$ where $K$ is some compact set. Denote by $L_{\varrho}$ the line bundle $L$ endowed with the trivialization @ outside some compact set.

With this understood, we will introduce a suitable analytic setting for the equation (3.1) following [K-M2].

The equation (3.1) for $s_{\omega}$ has the element $(\mathbb{I}, 0, d) \in \Gamma\left(\Lambda^{0,0}\right) \times \Gamma\left(\Lambda^{0,2}\right) \times \mathcal{A}(X \times \mathbb{C})$ as a special solution for any choice of $r$. (Here $X \times \mathbb{C}$ means the trivial line bundle over $X$.) We will adopt it as an asymptotic solution and define the function spaces $\Gamma_{0}, \mathcal{A}_{0}$ and $\mathcal{G}_{0}$ as follows:

$$
\begin{aligned}
& \Gamma_{0}:=\left\{(\alpha, \beta) \in \Gamma^{\infty}\left(\Lambda^{0,0}\left(L_{\varrho}\right) \oplus \Lambda^{0,2}\left(L_{\varrho}\right)\right) \mid(\alpha, \beta)-(\mathbb{I}, 0)\right.\text { has a compact support }\}, \\
& \mathcal{A}_{0}:=\left\{a \in \mathcal{A}^{\infty}\left(L_{\varrho}\right) \mid a-d \text { has a compact support }\right\} \\
& \mathcal{G}_{0}:=\left\{u \in C^{\infty}(X ; \mathbb{C})|| u \mid=1, u-1 \text { has a compact support }\right\} .
\end{aligned}
$$

$\varrho$ allows us to identify $\Lambda^{0,0}\left(L_{\varrho}\right) \oplus \Lambda^{0,2}\left(L_{\varrho}\right)$ with $\Lambda^{0,0} \oplus \Lambda^{0,2}$ outside some compact set $K$. Similary, $\left.a\right|_{X \backslash K}$ can be regarded as a U(1) connection of the trivial complex line bundle over $X \backslash K$. These identifications are implicit in (3.3). We will adopt some suitable completions of the spaces $\Gamma, \mathcal{A}$ and $\mathcal{G}$ as our function spaces.

$\mathcal{G}$ is the completion of $\mathcal{G}_{0}$ with respect to the Sobolev $W^{k+1,2}$ norm defined by the Riemannian metric $g_{J}$ and the covariant derivatives.

$\mathcal{A}_{0}$ can be identified with the space of compact support, smooth and pure imaginary self-dual 2 -forms by choosing a base point $a_{0}$. $\mathcal{A}$ is the completion of $\mathcal{A}_{0}$ with respect to the usual $W^{k, 2}$ norm for differential forms.

Define the Sobolev $W^{k, 2}$-norm for $\Gamma_{0}$ by making use of the Riemannian metric $g_{J}$, the Hermitian metric of $W_{s} \equiv \Lambda^{0,0}\left(L_{\varrho}\right) \oplus \Lambda^{0,2}\left(L_{\varrho}\right)$ and the covariant derivative $\nabla_{J}^{1} \otimes \mathrm{id}+\mathrm{id} \otimes \nabla_{a_{0}} . \Gamma$ is the completion of $\Gamma_{0}$ with respect to this norm.

Let us fix $k$ sufficiently large so that the Sobolev embedding theorem implies that these function spaces belong to $C^{1}$. Then the Sobolev multiplication theorem implies that the gauge group $\mathcal{G}$ acts naturally on $\Gamma \times \mathcal{A}$. In fact, these spaces are smooth Hilbert manifolds with the former acting as a Hilbert Lie group. Furthermore, the action is free. Thus, the quotient space $\mathcal{B}$ is also a Hilbert manifold. The standard argument in gauge theory shows that $\mathcal{B}$ is Hausdorff. See [K-M2].

To have the equation transverse, we introduce a Banach space $\mathcal{N}$ as a completion of the space of compact support, smooth, pure imaginary self-dual 2 -forms. The norm $\|\cdot\|_{\mathcal{N}}$ is given by $\|\eta\|_{\mathcal{N}}:=\left\|\exp \left(\epsilon_{1} \sigma\right) \cdot \eta\right\|_{C^{l}(X)}$ where $\epsilon_{1}>0$ and $l \geq k+1$ are fixed. We always assume that the $\eta$ in the equation (3.1) is chosen from this Banach space.

With this understood, we will give some results needed later and the definition of the monopole invariant in [K-M2] in the form suitable for our terminology.

Proposition 3.1 ( $\overline{\mathrm{K}-\mathrm{M} 2})$. Let $(\alpha, \beta, a) \in \Gamma \times \mathcal{A}$ be a solution of the equation (3.1). There exist positive constants $C_{0}$ and $C_{0}^{\prime}$ which depend only on $\left(\omega, J, c_{1}\left(L_{\varrho}\right)\right)$ 
and have the following significance: If $r \geq C_{0}$, then

$$
\begin{gathered}
\int_{X}\left|\nabla_{a} \alpha\right|^{2}+2\left|\tilde{\nabla}_{a} \beta\right|^{2}+\frac{r}{2}\left(1-|\alpha|^{2}\right)^{2}+\frac{r}{2}\left(1+2|\alpha|^{2}+|\beta|^{2}\right)|\beta|^{2} \\
\leq C_{0}^{\prime}\|\eta\|_{L^{1}(X)}+2 \pi\left\langle c_{1}\left(L_{\varrho}\right) \cup[\omega],[X]\right\rangle .
\end{gathered}
$$

Proposition $3.2([\mathrm{~K}-\mathrm{M} 2])$. Let $(\alpha, \beta, a) \in \Gamma \times \mathcal{A}$ be a solution of equations (3.1) with $r \geq 1$ and $\|\eta\|_{\mathcal{N}} \leq 1$. There exist positive constants $\nu_{r}$ and $\chi_{r}$ which depend only on $r$ and on $\left(\omega, J, c_{1}\left(L_{\varrho}\right)\right)$ and have the following significance:

$$
\left.\left\{\left.|| \alpha\right|^{2}-\left.1|+| \nabla_{a} \alpha\right|^{2}+|\beta|^{2}+\left|\tilde{\nabla}_{a} \beta\right|^{2}+\left|P^{+} F_{a}\right|+\left|P^{-} F_{a}\right|\right\}\right|_{x} \leq \chi_{r} e^{-\nu_{r} \sigma(x)}
$$

for any $x \in X$.

Theorem 3.3 ([K-M2] $)$. Define $\mathcal{M}(\omega, J, s, \varrho)$ to be the set

$$
\left\{([\alpha, \beta, a], \eta, r) \in \mathcal{B} \times \mathcal{N} \times \mathbb{R}^{>0} \mid(\alpha, \beta, a) \text { obeys (3.1) with these } \eta \text { and } r\right\},
$$

where $[(*)]$ means the gauge equivalence class of $(*)$. Then $\mathcal{M}(\omega, J, s, \rho)$ is a Banach submanifold of $\mathcal{B} \times \mathcal{N} \times \mathbb{R}^{>0}$. The projection $\operatorname{Pr}: \mathcal{M}(\omega, J, s, \rho) \mapsto \mathcal{N} \times \mathbb{R}^{>0}$ is a proper Fredholm map of index $\left\langle\left(c_{1}^{2}\left(L_{\varrho}\right)-c_{1}\left(L_{\varrho}\right) \cup c_{1}(K),[X]\right\rangle\right.$ and the index line bundle has a canonical orientation determined by $(\omega, J)$.

Here $c_{1}\left(L_{\varrho}\right)$ means $c_{1}(L, \tilde{\varrho}) . c_{1}(L, \tilde{\varrho})$ and $[X]$ are as explained in Section $1 . K$ denotes the canonical line bundle of $(X, J)$. Theorem 3.3 implies that if $(\eta, r)$ is generic, namely, if it is chosen from a suitable Baire subset of $\mathcal{N} \times \mathbb{R}^{>0}$, then $\operatorname{Pr}^{-1}(\eta, r)$ is a compact oriented manifold of dimension $\left\langle\left(c_{1}^{2}\left(L_{\varrho}\right)-c_{1}\left(L_{\varrho}\right) \cup\right.\right.$ $\left.c_{1}(K),[X]\right\rangle$. We will refer to $\operatorname{Pr}^{-1}(\eta, r)$ as the moduli space.

Definition 3.4. The monopole invariant $S W: S(X, \omega) \mapsto \mathbb{Z}$ is defined as follows:

(1) If $\left\langle\left(c_{1}^{2}\left(L_{\varrho}\right)-c_{1}\left(L_{\varrho}\right) \cup c_{1}(K),[X]\right\rangle \neq 0\right.$, then $S W(s, \varrho)=0$.

(2) If $\left\langle c_{1}^{2}\left(L_{\varrho}\right)-c_{1}\left(L_{\varrho}\right) \cup c_{1}(K),[X]\right\rangle=0$, then $S W(s, \varrho)$ is the sum of the suitable signs that are imposed to each connected component of the 0-dimensional manifold $\operatorname{Pr}^{-1}(\eta, r)$ for generic $(\eta, r)$. It does not depend on the choice of the pair $(\eta, r)$.

\section{The Statement of the Main Result}

Let $(X, \omega, J)$ be a weakly convex almost Kähler 4 -manifold. Let $\mathcal{M}(\omega, J, s, \rho)$ be the space as given in Section 3. Our main result follows:

Theorem 4.1. Let $\left\{r_{n}\right\}_{n \in \mathbb{N}}$ be a sequence of positive numbers which tends to infinity when $n$ tends to infinity. Suppose there exists a sequence $\left\{\left(\alpha_{n}, \beta_{n}, a_{n}, \eta_{n}\right)\right\}_{n \in \mathbb{N}}$ such that $\left(\left[\alpha_{n}, \beta_{n}, a_{n}\right], \eta_{n}, r_{n}\right) \in \mathcal{M}(\omega, J, s, \rho)$ obeying $\left\|\eta_{n}\right\|_{\mathcal{N}} \leq e^{-r_{n}}$. Then, after passing to a suitable subsequence, $\left\{\alpha_{n}^{-1}(0)\right\}_{n \in \mathbb{N}}$ converges in the Hausdorff topology to a compact $J$-holomorphic curve $D$ (which may have multiple irreducible components) whose homology class $[D] \in H_{2}(X, \mathbb{Z})$ is the Poincaré dual of $c_{1}\left(L_{\varrho}\right)$.

This theorem is an extension of the main result in [T1] where $X$ is supposed to be closed. When $X$ is non-compact, we must overcome the following problems:

The first one is that the sets $\alpha_{n}^{-1}(0)$ may possibly escape to the infinity of the end when $n$ tends to infinity. The monotonicity formula for local energy integral can settle this problem as long as we have an a priori bound for the total energy integral $\left.\frac{1}{4} \int_{X} r|1-| \alpha\right|^{2} \mid$, the bound which is independent of $r$. 
The second one is that it is not obvious at first whether the a priori bound for the total energy integral does exist.

The third one, which is related to the second one, is that the argument in [T1] to find the a priori $C^{0}$ bound of the anti-self-dual part of the curvature does not work directly in our case.

Our strategy is divided into 3 steps:

Step 1. We will show in Sections 5 and 6 that the $C^{0}$ estimates of the terms $\left.\left.|1-| \alpha\right|^{2}|,| \beta\right|^{2},\left|\nabla_{a} \alpha\right|^{2},\left|\tilde{\nabla}_{a} \beta\right|^{2}$ and $\left|F_{a}^{ \pm}\right|^{2}$ given in [T1] are also valid in our case. The major difference from [T1] is in the proof of the $C^{0}$ estimate for $F_{a}^{-}$, which is given in Section 6 .

Step 2. We will derive in Section 7 an a priori estimate of the total energy integral.

Step 3. We will derive in Section 8 a slightly refined monotonicity formula for local energy integral.

With these achieved, we can easily show that $\alpha_{n}^{-1}(0)$ does remain in some compact set when $n$ tends to infinity. This will be done in Section 9 and allows us to handle the issue as if our manifold $X$ were compact. Thus applying the arguments in [T1] almost directly, we can prove Theorem 4.1.

Before going on to the proof, let us agree that we are subject to Assumption 1 and Conventions 1 and 2 below in Section 5, 6, 7, 8 and 9 unless otherwise specified:

Assumption 1. We suppose that $r \geq 1$ and that

$$
\|\eta\|_{\mathcal{N}} \leq e^{-r}
$$

Convention 1. We adopt the following convention for constants:

a. The symbol $C$ with no subscript stands for a positive constant which depends only on the data $\left(\omega, J, c_{1}\left(L_{\varrho}\right)\right)$ and that the value which $C$ is supposed to be may vary from line to line even in a single formula.

b. The symbol $C$ with some subscript such as $C_{1}$ stands for a positive constant which depends only on $\left(\omega, J, c_{1}\left(L_{\varrho}\right)\right)$ and the value which it is supposed to be is consistent in later arguments.

Convention 2. If we say that a constant, such as $r, \kappa$ and so on, is sufficiently large, it means that it is larger than a suitable positive constant that depends only on $\left(\omega, J, c_{1}\left(L_{\varrho}\right)\right)$.

\section{Preliminary estimates}

We will devote this section to derive preliminary estimates.

It is known that the Dolbeaut operators on an almost Kähler manifold satisfy the Kähler identities. See [Ma]. Our starting point is the following identities which derive from the Kähler identities after a short calculation (see [Ko] ):

$$
\begin{aligned}
& \bar{\partial}_{a}^{*} \bar{\partial}_{a} \alpha=\frac{1}{2} \nabla_{a}^{*} \nabla_{a} \alpha-\frac{1}{2} \sqrt{-1}\left(\Lambda F_{a}\right) \alpha, \\
& \bar{\partial}_{a} \bar{\partial}_{a} \alpha=N \circ \partial_{a} \alpha+F_{a}^{0,2} \alpha, \\
& \bar{\partial}_{a}^{*} \bar{\partial}_{a}^{*} \beta=\partial_{a}^{*} \circ N^{*} \beta+\left(F_{a}^{0,2}\right)^{*} \beta, \\
& \bar{\partial}_{a} \bar{\partial}_{a}^{*} \beta=\frac{1}{2} \tilde{\nabla}_{a}^{*} \tilde{\nabla}_{a} \beta+\frac{1}{2} \sqrt{-1}\left(\Lambda\left(F_{a}+F_{\bar{K}}\right)\right) \beta,
\end{aligned}
$$


where $N \in \operatorname{Hom}\left(\Lambda^{1,0}, \Lambda^{0,2}\right)$ is the Nijenhuis tensor of $J, \tilde{\nabla}_{a}$ is the unitary connection of $\Lambda^{0,2} \otimes L$ whose $(1,0)$ part agrees with the Dolbeaut operator $\partial_{a}: \Omega^{0,2}(L) \mapsto$ $\Omega^{1,2}(L)$ and $F_{a}+F_{\bar{K}}$ is the cuvature of $\tilde{\nabla}_{a}$.

Remark. In the case where $(\omega, J)$ is Kähler, the identities (5.1) are exactly the Weitzenböck formula of a Dirac operator.

It follows from (3.1) and (5.1) after a short calculation that

$$
\begin{aligned}
\frac{1}{2} \nabla_{a}^{*} \nabla_{a} \alpha= & -\frac{r}{8}\left(-1+|\alpha|^{2}+|\beta|^{2}\right) \alpha-\partial_{a}^{*} \circ N^{*} \beta \\
& -\left(\eta^{0,2}\right)^{*} \beta+\frac{\sqrt{-1}}{2}(\Lambda \eta) \alpha, \\
\frac{1}{2} \tilde{\nabla}_{a}^{*} \tilde{\nabla}_{a} \beta=- & \frac{r}{8}\left(+1+|\alpha|^{2}+|\beta|^{2}\right) \beta+N \circ \partial_{a} \alpha \\
& -\frac{\sqrt{-1}}{2}\left(\Lambda F_{\bar{K}}\right) \beta-\alpha \eta^{0,2}-\frac{\sqrt{-1}}{2}(\Lambda \eta) \beta .
\end{aligned}
$$

Taking the inner product of (5.2.1) with $\alpha$ and making use of the identity $\frac{1}{2} \triangle\left(|\alpha|^{2}\right)$ $=\left\langle\nabla_{a}^{*} \nabla_{a}, \alpha\right\rangle-\left|\nabla_{a} \alpha\right|^{2}$, it follows that

$$
\begin{aligned}
\frac{1}{4} \triangle\left(|\alpha|^{2}\right)= & -\frac{1}{2}\left|\nabla_{a} \alpha\right|^{2}-\frac{r}{8}\left(-1+|\alpha|^{2}+|\beta|^{2}\right)|\alpha|^{2}-\left\langle\partial_{a}^{*} \circ N^{*} \beta, \alpha\right\rangle \\
& -\left\langle\left(\eta^{0,2}\right)^{*} \beta, \alpha\right\rangle+\frac{\sqrt{-1}}{2} \Lambda \eta|\alpha|^{2} .
\end{aligned}
$$

Similarly, it follows that

$$
\begin{aligned}
\frac{1}{4} \triangle\left(|\beta|^{2}\right)= & -\frac{1}{2}\left|\tilde{\nabla}_{a} \beta\right|^{2}-\frac{r}{8}\left(1+|\alpha|^{2}+|\beta|^{2}\right)|\beta|^{2} \\
& +\left\langle\beta, N \circ \partial_{a} \alpha\right\rangle-\frac{\sqrt{-1}}{2}\left(\Lambda F_{\bar{K}}\right)|\beta|^{2} \\
& -\left\langle\eta^{0,2} \alpha, \beta\right\rangle-\frac{\sqrt{-1}}{2}(\Lambda \eta)|\beta|^{2} .
\end{aligned}
$$

Since $(X, \omega, J)$ is weakly convex, $N, F_{\bar{K}}$ and their higher covariant derivatives are all bounded. Thus by dropping some non-positive terms and applying Schwarz' inequality, we obtain

Lemma 5.0. Let $(\alpha, \beta, a)$ be a solution of equations (3.1) with $r \geq 1$. It holds that

$$
\begin{aligned}
\left(\frac{1}{2} \triangle+\frac{r}{4}|\alpha|^{2}\right)\left(|\alpha|^{2}-1\right) & \\
\leq-\left|\nabla_{a} \alpha\right|^{2} & +C\left|\tilde{\nabla}_{a} \beta\right| \cdot|\alpha|+(C+|\eta|)|\alpha| \cdot|\beta|+|\eta| \cdot|\alpha|^{2}, \\
\left(\frac{1}{2} \triangle+\frac{r}{4}|\alpha|^{2}\right)|\beta|^{2} \leq & -\left|\tilde{\nabla}_{a} \beta\right|^{2}-\frac{r}{4}\left(1-\frac{C}{r}\right)|\beta|^{2} \\
& +C\left|\nabla_{a} \alpha\right| \cdot|\beta|+|\eta| \cdot|\alpha| \cdot|\beta|+|\eta| \cdot|\beta|^{2} .
\end{aligned}
$$

By making use of it, we can show

Proposition 5.1. Let $(\alpha, \beta, a)$ be a solution of equations (3.1) with $r \geq 1$. There exist non-negative constants $\kappa_{1}, \kappa_{2}$ which depend only on $\left(\omega, J, c_{1}\left(L_{\varrho}\right)\right)$ and have 
the following significance: If $p$ and $\zeta$ obey that $1 \geq p \geq 0, \zeta>0$, then

$$
\begin{aligned}
\left(\frac{1}{2} \triangle+\frac{r}{4}|\alpha|^{2}\right) & \left\{|\alpha|^{2}-1+\zeta r^{p}|\beta|^{2}\right\} \\
\leq & -\left(1-\frac{\zeta \kappa_{1}}{r^{1-p}}\right)\left|\nabla_{a} \alpha\right|^{2}-\frac{\zeta}{2} r^{p}\left|\tilde{\nabla}_{a} \beta\right|^{2}-\frac{\zeta}{24} r^{1+p}|\beta|^{2}+\kappa_{2}\left(\frac{1}{\zeta}+\zeta\right) \frac{1}{r^{p}}|\alpha|^{2} .
\end{aligned}
$$

Proof. It follows from (4.1), (5.4.1), (5.4.2) and Hölder's inequality that

$$
\begin{aligned}
\text { R.H.S. } & \leq-\left|\nabla_{a} \alpha\right|^{2}-\zeta r^{p}\left|\tilde{\nabla}_{a} \beta\right|^{2}-\frac{\zeta r^{1+p}}{8}|\beta|^{2} \\
& +C_{1} \zeta r^{p}\left|\nabla_{a} \alpha\right||\beta|+C_{2}\left|\tilde{\nabla}_{a} \beta\right||\alpha|+C_{3}(1+\zeta)|\alpha||\beta|+|\eta||\alpha|^{2} \\
& \leq-\left(1-\frac{C_{1} \zeta \epsilon_{1}}{2 r^{1-p}}\right)\left|\nabla_{a} \alpha\right|^{2}-r^{p}\left(\zeta-\frac{C_{2} \epsilon_{2}}{2}\right)\left|\tilde{\nabla}_{a} \beta\right|^{2} \\
& -\frac{r^{1+p}}{8}\left(\zeta-\frac{4 C_{1} \zeta}{\epsilon_{1}}-\frac{4 C_{3}}{\epsilon_{3}}(1+\zeta)\right)|\beta|^{2}+\frac{1}{r^{p}}\left(\frac{C_{2}}{2 \epsilon_{2}}+\frac{C_{3} \epsilon_{3}}{2 r}(1+\zeta)+r^{p}|\eta|\right)|\alpha|^{2} .
\end{aligned}
$$

By putting $\epsilon_{1}=12 C_{1}, \epsilon_{2}=\frac{\zeta}{C_{2}}$ and $\epsilon_{3}=\frac{12 C_{3}(1+\zeta)}{\zeta}$, we obtain the result.

Proposition 5.2. Let $(\alpha, \beta, a)$ be a solution of equations (3.1) with $r \geq 1$. There exists a constant $C$ which depends only on $\left(\omega, J, c_{1}\left(L_{\varrho}\right)\right)$ and has the following significance: It holds that

$$
|\alpha|^{2}+|\beta|^{2} \leq 1+\frac{C}{r}
$$

Proof. Define $f$ to be $|\alpha|^{2}-1+|\beta|^{2}-\frac{\kappa}{r}$ where $\kappa$ is a positive constant determined later. Proposition 5.1 implies that $f$ obeys

$$
\left(\frac{1}{2} \triangle+\frac{r}{4}|\alpha|^{2}\right) f \leq\left(C-\frac{\kappa}{4}\right)|\alpha|^{2} .
$$

By taking $\kappa$ sufficiently large, the right-hand side is nonpositive. On the other hand, $f$ is negative outside some compact set (that may depend on $r$ and $\kappa$ ). Thus a maximum principle implies $f \leq 0$.

Proposition 5.3. Let $(\alpha, \beta, a)$ be a solution of equations (3.1) with $r \geq 1$. There exists a constant $C_{M}$ which depends only on $\left(\omega, J, c_{1}\left(L_{\varrho}\right)\right)$ and has the following significance: It holds that

$$
|\beta|^{2} \leq \frac{1}{r}\left(1-|\alpha|^{2}\right)+\frac{C_{M}}{r^{3}}
$$

Proof. Let $\zeta_{1}$ be a fixed positive constant such that $\zeta_{1} \kappa_{1} \leq 1$. Define $f$ to be $|\alpha|^{2}-1+\zeta_{1} r|\beta|-\frac{\kappa}{r^{2}}$ where $\kappa$ is a positive constant determined later. Proposition 5.1 implies that $\left(\frac{1}{2} \triangle+\frac{r}{4}|\alpha|^{2}\right) f \leq\left(C-\frac{\kappa}{4}\right) \frac{1}{r}|\alpha|^{2}$. Thus if $\kappa$ is sufficiently large, the same argument as in the proof of Proposition 5.2 implies $f \leq 0$.

Proposition 5.4. Let $(\alpha, \beta, a)$ be a solution of equations (3.1) with $r \geq 1$. There exist positive constants $\mu_{1}, \mu_{2}$ which depend only on $\left(\omega, J, c_{1}\left(L_{\varrho}\right)\right)$ and have the following significance: It holds that

$$
\left|F_{a}^{+}\right| \leq \frac{r}{4 \sqrt{2}}\left(1+\frac{\mu_{1}}{r}\right)\left(1-|\alpha|^{2}\right)+\frac{\mu_{2}}{r} .
$$

Proof. This follows directly from (3.1.2), (3.1.3) and Proposition 5.3. 
6. The $C^{0}$ Estimate of the ANTi-SElf-Dual Part of the CURVAture

We will devote the whole of this section to prove

Proposition 6.1. Let $(\alpha, \beta, a)$ be a solution of equations (3.1) with $r \geq 1$. There exist non-negative constants $\mu_{3}, \mu_{4}$ which depend only on $\left(\omega, J, c_{1}\left(L_{\varrho}\right)\right)$ and have the following significance: It holds that

$$
\left|F_{a}^{-}\right| \leq \frac{r}{4 \sqrt{2}}\left(1+\frac{\mu_{3}}{r^{\frac{1}{4}}}\right)\left(1-|\alpha|^{2}\right)+\frac{\mu_{4}}{r^{\frac{1}{4}}} .
$$

This estimate will be needed in the proofs of Proposition 7.1 and Proposition 8.1 .

The proof is divided into 8 steps.

Step (0). Denote $\left|F_{a}^{-}\right|$by $t$. We will derive a differential inequality that $t$ obeys.

Lemma 6.2. $t$ obeys the following inequality on $X \backslash t^{-1}(0)$ :

$$
\left(\frac{1}{2} \triangle+\frac{r}{4}|\alpha|^{2}\right) t \leq R t+\frac{r}{4 \sqrt{2}}\left(\left|\nabla_{a} \alpha\right|^{2}+\left|\tilde{\nabla}_{a} \beta\right|^{2}\right)+C r|\beta|^{2}+\left|h_{\eta}\right| .
$$

Here $R$ is a non-negative function derived from the Riemannian metric and $h_{\eta}$ denotes $\frac{1}{4} P^{-}\left(d^{*} d \eta\right)$ where $P^{-}$stands for the orthogonal projection $P^{-}: \stackrel{2}{\Lambda} \mapsto \Lambda^{+}$.

Proof. The Bianchi identity implies that

$$
d F_{a}^{+}+d F_{a}^{-}=0 .
$$

Then a Bochner-Weitzenbock formula implies that

$$
\frac{1}{2} \nabla^{*} \nabla F_{a}^{-}+\mathcal{R} F_{a}^{-}=-P^{-} d^{*} d F_{a}^{+},
$$

where $\mathcal{R} \in \operatorname{Hom}\left(\Lambda^{-}, \Lambda^{-}\right)$derives from the anti-self-dual part of the curvature of the Riemannian metric and the scalar curvature. (3.1.2) and (3.1.3) imply that

$$
\begin{aligned}
& \text { R.H.S. of }(6.4)=P^{-} d^{*} d\left\{-\frac{\sqrt{-1} r}{8}\right.\left(-1+|\alpha|^{2}-|\beta|^{2}\right) \\
&\left.\omega-\frac{r}{4} \alpha^{*} \beta+\frac{r}{4} \alpha \beta^{*}-\eta\right\} .
\end{aligned}
$$

By making use of the Kähler identities,

$$
\begin{aligned}
& P^{-} d^{*} d\left\{-\frac{\sqrt{-1} r}{8}\left(-1+|\alpha|^{2}-|\beta|^{2}\right) \omega\right\} \\
= & -\frac{\sqrt{-1} r}{8} P^{-}\left(\partial^{*} \partial+\bar{\partial}^{*} \bar{\partial}\right)\left(|\alpha|^{2}-|\beta|^{2}\right) \omega \\
= & -\frac{\sqrt{-1} r}{8} P^{-}(-\sqrt{-1 \partial} \Lambda \partial+\sqrt{-1} \partial \Lambda \bar{\partial})\left(|\alpha|^{2}-|\beta|^{2}\right) \omega \\
& \left(\text { since Image }\left(\Lambda: \Lambda^{2,2} \mapsto \Lambda^{1,1}\right) \subset \Lambda^{+}\right) \\
= & -\frac{r}{4} P^{-}\left(\bar{\partial} \partial|\alpha|^{2}+\partial \bar{\partial}|\beta|^{2}\right) \\
& \left(\text { since } \Lambda \circ(\omega \wedge)=i d \text { on } \stackrel{1}{\Lambda} \text { and since } \bar{\partial} \partial+\partial \bar{\partial}=0 \text { on } \Lambda^{0,0}\right) .
\end{aligned}
$$

Thus we obtain the equality (6.7) below:

R.H.S. of $(6.6)=-\frac{r}{4} P^{-}\left\{\left\langle\bar{\partial}_{a} \partial_{a} \alpha, \alpha\right\rangle_{L}+\left\langle\alpha, \partial_{a} \bar{\partial}_{a} \alpha\right\rangle_{L}-\left\langle\partial_{a} \alpha, \partial_{a} \alpha\right\rangle_{L}+\left\langle\bar{\partial}_{a} \alpha, \bar{\partial}_{a} \alpha\right\rangle_{L}\right.$ $\left.+\left\langle\partial_{\tilde{\nabla}_{a}} \bar{\partial}_{\tilde{\nabla}_{a}} \beta, \beta\right\rangle_{L \otimes \bar{K}}+\left\langle\beta, \bar{\partial}_{\tilde{\nabla}_{a}} \partial_{\tilde{\nabla}_{a}} \beta\right\rangle_{L \otimes \bar{K}}+\left\langle\partial_{\tilde{\nabla}_{a}} \beta, \partial_{\tilde{\nabla}_{a}} \beta\right\rangle_{L \otimes \bar{K}}-\left\langle\bar{\partial}_{\tilde{\nabla}_{a}} \beta, \bar{\partial}_{\tilde{\nabla}_{a}} \beta\right\rangle_{L \otimes \bar{K}}\right\}$ 
where $\langle\cdot, \cdot\rangle_{L}$ and $\langle\cdot, \cdot\rangle_{L \otimes \bar{K}}$ are the Hermitian inner products of the line bundle $L$ and $L \otimes \bar{K}$, respectively. Further, the exterior products of the forms are implicit in these expressions. $\partial_{\tilde{\nabla}_{a}}$ stands for the coupled Dolbeaut operator $\partial_{\tilde{\nabla}_{a}}: \Omega^{p, q}(L \otimes \bar{K}) \mapsto$ $\Omega^{p+1, q}(L \otimes \bar{K})$.

On the other hand, since $\alpha^{*} \beta$ is a $(0,2)$ form, it follows that

$$
\begin{aligned}
P^{-} d^{*} d\left(-\frac{1}{4} \alpha^{*} \beta\right) & =\frac{1}{4} P^{-}(\sqrt{-1} \partial \Lambda \partial)\left(\alpha^{*} \beta\right) \\
& =\frac{\sqrt{-1}}{4} P^{-} \partial\left\{\Lambda\left(\bar{\partial}_{a} \alpha\right)^{*} \wedge \beta+\Lambda \alpha^{*} \partial \beta\right\} \\
& =-\frac{\sqrt{-1}}{4} P^{-} \partial\left\{-\Lambda\left(\bar{\partial}_{a}^{*} \beta\right)^{*} \wedge \beta+\sqrt{-1}\left(\alpha^{*} \bar{\partial}_{a}^{*} \beta\right)\right\} \\
& =-\frac{\sqrt{-1}}{4} P^{-} \partial\left\{-\Lambda\left(\bar{\partial}_{a}^{*} \beta\right)^{*} \wedge \beta-\sqrt{-1} \alpha^{*}\left(\bar{\partial}_{a} \alpha\right)\right\}
\end{aligned}
$$

where we have used (3.1.1) and the Kähler identities. Applying to (6.8) the identity $\sqrt{-1} \Lambda\left(\beta \wedge\left(\bar{\partial}_{a}^{*} \beta\right)^{*}\right) \equiv \beta\left(\partial_{\tilde{\nabla}_{a}} \beta\right)^{*}$ which also derives from the Kähler identities, we obtain

$$
P^{-} d^{*} d\left(-\frac{1}{4} \alpha^{*} \beta\right)=-\frac{1}{4} P^{-} \partial\left\{-\left\langle\beta, \partial_{\tilde{\nabla}_{a}} \beta\right\rangle_{L \otimes \bar{K}}+\left\langle\bar{\partial}_{a} \alpha, \alpha\right\rangle_{L}\right\}
$$

Taking its complex conjugate, we obtain the equality

$$
P^{-} d^{*} d\left(\frac{1}{4} \alpha \beta^{*}\right)=-\frac{1}{4} P^{-} \bar{\partial}\left\{\left\langle\partial_{\tilde{\nabla}_{a}} \beta, \beta\right\rangle_{L \otimes \bar{K}}-\left\langle\alpha, \bar{\partial}_{a} \alpha\right\rangle_{L}\right\} .
$$

Therefore, by applying the identities

$$
\begin{aligned}
P^{-} F_{a} & \equiv P^{-}\left(\bar{\partial}_{a} \partial_{a}+\partial_{a} \bar{\partial}_{a}\right), \\
P^{-}\left(F_{a}+F_{\bar{K}}\right) & \equiv P^{-}\left(\bar{\partial}_{\tilde{\nabla}_{a}} \partial_{\tilde{\nabla}_{a}}+\partial_{\tilde{\nabla}_{a}} \bar{\partial}_{\tilde{\nabla}_{a}}\right)
\end{aligned}
$$

to $(6.4),(6.7)$ and (6.9) and summing up the result, it follows that

$$
\begin{gathered}
\frac{1}{2} \nabla \nabla^{*} F_{a}^{-}+\mathcal{R} F_{a}^{-}=-\frac{r}{4}\left\langle P^{-} F_{a} \alpha, \alpha\right\rangle_{L}-\frac{r}{4}\left\langle P^{-}\left(F_{a}+F_{\bar{K}}\right) \beta, \beta\right\rangle_{L \otimes \bar{K}} \\
+\frac{r}{4} P^{-}\left\{\left\langle d_{a} \alpha, d_{a} \alpha\right\rangle_{L}+\left\langle d_{\tilde{\nabla}_{a}} \beta, d_{\tilde{\nabla}_{a}} \beta\right\rangle_{L \otimes \bar{K}}\right\}+\frac{1}{4} P^{-}\left(d^{*} d \eta\right) .
\end{gathered}
$$

By taking the Hermitian inner product of this with $P^{-} F_{a}$, and making use of the inequlity $(\triangle|F|)|F| \leq\left\langle\nabla^{*} \nabla F, F\right\rangle$ for an arbitrary non-vanishing real 2-form $F$, we obtain the inequality

$$
\begin{aligned}
\left(\frac{1}{2} \triangle+\frac{r}{4}|\alpha|^{2}\right) t \leq & |\mathcal{R}| t+\frac{r}{4}|\beta|^{2}\left|F_{\bar{K}}\right|+\frac{r}{4}\left|P^{-}\left\{\left\langle d_{a} \alpha, d_{a} \alpha\right\rangle_{L}+\left\langle d_{\tilde{\nabla}_{a}} \beta, d_{\tilde{\nabla}_{a}} \beta\right\rangle_{L \otimes \bar{K}}\right\}\right| \\
& +\frac{1}{4}\left|P^{-} d^{*} d \eta\right| \\
\leq & |\mathcal{R}| t+C r|\beta|^{2}+\frac{r}{4 \sqrt{2}}\left(\left|\nabla_{a} \alpha\right|^{2}+\left|\tilde{\nabla}_{a} \beta\right|^{2}\right)+\left|h_{\eta}\right| .
\end{aligned}
$$

Step (1). We will introduce a comparison function $q_{0}$.

Take a sufficiently large $\kappa>1$ and define the function $q_{0}$ by

$$
q_{0}:=\frac{r}{4 \sqrt{2}}\left(1+\frac{2 \kappa_{1}}{r^{\frac{1}{2}}}\right)\left(1-|\alpha|^{2}-r^{\frac{1}{2}}|\beta|^{2}+\frac{\kappa}{r^{\frac{3}{2}}}\right) .
$$


Lemma 6.2, Proposition 5.1 and Proposition 5.3 imply that if $r$ is sufficiently large, $q_{0}$ is positive and obeys

$$
\left(\frac{1}{2} \triangle+\frac{r}{4}|\alpha|^{2}\right)\left(t-q_{0}\right) \leq R_{0} \cdot t+\left|h_{\eta}\right|
$$

where $R_{0}$ denotes $\sup _{X}|\mathcal{R}|$.

Here we applied Proposition 5.1 to $p=\frac{1}{2}$ and $\zeta=1$ and used the fact that $1 \leq$ $\left(1-\frac{\kappa_{1}}{r^{\frac{1}{2}}}\right)\left(1+\frac{2 \kappa_{1}}{r^{\frac{1}{2}}}\right)$ if $r$ is sufficiently large. We included the constant term $\kappa r^{-\frac{3}{2}}$ in the definition of $q_{0}$ in order to compensate for the last term of the R.H.S. in the inequality of Proposition 5.1, whose existence derives from the Nijenhuis tensor of $J$.

Step (2). We will define a good comparison function $q \in W_{0}^{2,2}(X)$ so that $q$ obeys $t \leq q_{0}+q$.

Lemma 6.3. The operator $\left(\frac{1}{2} \triangle+\frac{r}{4}|\alpha|^{2}\right): C_{0}^{\infty}(X) \mapsto C_{0}^{\infty}(X)$ extends to a selfadjoint operator $\tilde{L}$ over $L^{2}(X)$ with $\operatorname{Dom}(\tilde{L})=W_{0}^{2,2}(X)$. Further, $\tilde{L}$ is surjective.

Here $W_{0}^{2,2}(X)$ denotes the completion of $C_{0}^{\infty}(X)$ with respect to the Sobolev norm $\|*\|_{W^{2.2}(X)}$ defined by $\|f\|_{W^{2,2}(X)}:=\|f\|_{L^{2}(X)}+\|\nabla f\|_{L^{2}(X)}+\|\nabla \nabla f\|_{L^{2}(X)}$. We will give its proof in Appendix.

Define $q \in W_{0}^{2,2}(X)$ to be the unique solution of the equation

$$
\left(\frac{1}{2} \triangle+\frac{r}{4}|\alpha|^{2}\right) q=R_{0} \cdot t+h
$$

Here $h$ denotes $\left|h_{\eta}\right|$. By the construction, $h$ obeys $h \leq C e^{-r}$ and decays like the function $e^{-\epsilon_{1} \sigma}$.

Lemma 6.4. $q$ obeys the following:

1. $q$ tends to zero uniformly at the end of $X$.

2. $q \in C^{2, \frac{1}{2}}(X)$.

3. $q \geq 0$.

Then a maximum principle applied to (6.14) and (6.15) implies that

$$
t \leq q_{0}+q .
$$

Proof of Lemma 6.4. Let $l>0$ denote $\min _{X} \sigma$. The property (A) of the Riemannian metric $g_{J}$ means that the geometries $\left\{\left.g_{J}\right|_{B(x, l)}\right\}_{x \in X}$ are bounded. Thus it follows from the standard $L^{p}$-theory of elliptic operators and the Sobolev embedding theorem that there exists a positive constant $C$ such that

$$
\|q\|_{C^{0}\left(B\left(x, \frac{l}{2}\right)\right)} \leq C\left(\|q\|_{L^{2}(B(x, l))}+\left\|R_{0} \cdot t+h\right\|_{C^{0}(B(x, l))}\right) .
$$

Then the first assertion follows from the fact that $\left\|R_{0} \cdot t+h\right\|_{C^{0}(B(x, l))}$ tends to zero uniformly when $\sigma(x)$ tends to infinity. The second assertion follows from the standard Hölder theory of elliptic operators since the right-hand side of (6.15) is in $C^{\frac{1}{2}}(X)$. Then a maximum principle verifies the third assertion.

Step (3). We will estimate $\|q\|_{L^{2}(X)}$ in terms of $\sup q$.

Taking the multiple of (6.15) with $q$ and adding $\underset{\frac{r}{4}}{\frac{X}{4}}\left(1-|\alpha|^{2}\right) q^{2}$ to both sides of it, we obtain the equality

$$
\frac{1}{4} \triangle\left(q^{2}\right)+\frac{1}{2}|\nabla q|^{2}+\frac{r}{4}|q|^{2}=\left(R_{0} \cdot t+h\right) q+\frac{r}{4}\left(1-|\alpha|^{2}\right) q^{2} .
$$


Applying Hölder's inequality to the both terms of the right-hand side, we see that it is no more than $\frac{3}{r}\left(R_{0} \cdot t+h\right)^{2}+\frac{3}{16} r\left(1-|\alpha|^{2}\right)^{2}\left(\sup _{X} q\right)^{2}+\frac{r}{6}|q|^{2}$. Thus it follows that

$$
\frac{1}{4} \triangle\left(q^{2}\right)+\frac{r}{12}|q|^{2} \leq \frac{6}{r}\left(R_{0}^{2} \cdot t^{2}+h^{2}\right)+\frac{3}{16} r\left(1-|\alpha|^{2}\right)^{2}\left(\sup _{X} q\right)^{2} .
$$

The very definition of $W_{0}^{2,2}(X)$ immediately implies that $\int_{X} \triangle\left(q^{2}\right) d \operatorname{vol}_{X}=0$. Thus by integrating (6.19) over $X$, we obtain the following inequality:

$$
\int_{X}|q|^{2} \leq C\left\{\frac{1}{r^{2}}\left(\int_{X} t^{2}\right)+\frac{e^{-2 r}}{r^{2}}+\frac{1}{r}\left(\sup _{X} q\right)^{2}\left(\int_{X} r\left(1-|\alpha|^{2}\right)^{2}\right)\right\} .
$$

On the other hand, Proposition 3.1 implies that

$$
\int_{X} r\left(1-|\alpha|^{2}\right)^{2} \leq C
$$

Further, it holds that

$$
\int_{X} t^{2} \leq C r+C
$$

In fact, the Chern-Weil theory implies that

$$
\int_{X} t^{2}=\int_{X}\left|F_{a}^{-}\right|^{2}=\int_{X}\left|F_{a}^{+}\right|^{2}-\left\langle c_{1}^{2}(L),[X]\right\rangle .
$$

On the other hand, (3.1.2), (3.1.3) and Proposition 3.1 imply that

$$
\int_{X}\left|F_{a}^{+}\right|^{2}=\frac{r}{32} \int_{X} r\left\{\left(1-|\alpha|^{2}-|\beta|^{2}\right)^{2}+2|\beta|^{2}\right\} \leq C r .
$$

Combining (6.20), (6.21) and (6.22) together, we obtain

$$
\|q\|_{L^{2}(X)} \leq \frac{C}{r^{\frac{1}{2}}}+\frac{C}{r^{\frac{1}{2}}} \sup _{X} q .
$$

Step (4). We will introduce a comparison function $p \in W_{0}^{2,2}(X)$. Define $p \in W_{0}^{2,2}(X)$ to be the unique solution of the equation

$$
\left(\frac{1}{2} \triangle+\frac{r}{4}|\alpha|^{2}\right) p=\left(R_{0} \cdot t+h\right)|\alpha|^{2} .
$$

Exactly the same way as in the case of Lemma 6.4, we can prove

Lemma 6.5. $p$ obeys the following:

(1) $p$ tends to zero uniformly at the end of $X$.

(2) $p \in C^{2, \frac{1}{2}}(X)$.

(3) $p \geq 0$.

Applying a maximum principle to $p-\frac{4}{r}\left(R_{0} \sup _{X} t+\sup _{X} h\right)$, it follows that

$$
\sup _{X} p \leq \frac{4}{r}\left(R_{0} \sup _{X} t+\sup _{X} h\right) .
$$

Step (5). We will estimate the upper bounds of both $q$ and $t$. We consider the equality below obtained from (6.15) and (6.26):

$$
\left(\frac{1}{2} \triangle+\frac{r}{4}|\alpha|^{2}\right)(q-p)=\left(R_{0} \cdot t+h\right)\left(1-|\alpha|^{2}\right) .
$$


We will apply to it the maximum principle of Gilbarg and Trudinger derived from Alexandrov and Bakel'man. See Theorem 9.20 in G-T.

Theorem 6.6 ([G-T] $)$. Let $D:=-a^{i j} \frac{\partial}{\partial x_{i}} \frac{\partial}{\partial x_{j}}+b^{j} \frac{\partial}{\partial x_{j}}+c$ be an elliptic operator defined on the unit ball in $\mathbb{R}^{4}$ and satisfy the conditions below:

(1) Let $A$ be a symmetric matrix $\left[a^{i j}\right]_{i j}$. There exist positive constants $\lambda_{1} \geq \lambda_{2}$ such that $\lambda_{1}|\xi|^{2} \geq \xi^{t} A \xi \geq \lambda_{2}|\xi|^{2}$ for all $\xi \in \mathbb{R}^{4}$. In other words, $A$ is uniformly positive definite.

(2) There exists $\lambda_{3} \geq 0$ such that $|b| \leq \lambda_{3}$ and $c \geq-\lambda_{3}$.

Then there exists a positive constant $C^{\prime}$ which depends only on $\lambda_{1}, \lambda_{2}$ and $\lambda_{3}$ and has the following significance: If $f \in C^{2}\left(\bar{B}_{1}\right)$ obeys the differential inequality $D f \leq g$ on $B_{1}^{+} \subset B_{1}$, then it follows that

$$
\sup _{B_{\frac{1}{2}}} f \leq C^{\prime}\left(\|f\|_{L^{2}\left(B_{1}^{+}\right)}+\|g\|_{L^{4}\left(B_{1}^{+}\right)}\right)
$$

where $B_{1}^{+}$denotes the subset $\left\{x \in B_{1} \mid f(x) \geq 0\right\}$.

The important point is that the coefficient $c$ is required to be bounded only from below. In $\left[\mathrm{G}-\mathrm{T}\right.$, the assumption of the statement requires that $|c| \leq \lambda_{3}$. But looking closely at the proof, we can easily see that this condition can be relaxed as above.

Let $x \in X$ attain the maximum of $q-p$. Theorem 6.6 is applied to (6.28) to show that

$$
\begin{aligned}
& \sup _{X}(q-p)=\sup _{B_{\frac{1}{2}}(x)}(q-p) \leq C\left\{\|q\|_{L^{2}(X)}+\|p\|_{L^{2}\left(B_{1}\left(x_{n}\right)\right)}\right. \\
& \left.+\left(\sup _{X} t+e^{-r}\right)\left\|1-|\alpha|^{2}\right\|_{L^{4}(X)}\right\} .
\end{aligned}
$$

The right-hand side of (6.29) is bounded from above by

$$
C\left(\frac{1}{r^{\frac{1}{2}}}+\frac{1}{r^{\frac{1}{2}}} \sup _{X} q+\frac{1}{r^{\frac{1}{4}}} \sup _{X} t\right)
$$

because of $(6.25),(6.27)$ and the inequality

$$
\int_{X}\left(1-|\alpha|^{2}\right)^{4} \leq C \int_{X}\left(1-|\alpha|^{2}\right)^{2} \leq \frac{C}{r}
$$

that derives from Proposition 5.2 and (6.21). On the other hand, the left-hand side of (6.29) is bounded from below by

$$
\sup _{X} q-\frac{C}{r} \sup _{X} t-C \frac{e^{-r}}{r} .
$$

Therefore, for sufficiently large $r$, it follows that

$$
\sup _{X} q \leq C\left(\frac{1}{r^{\frac{1}{2}}}+\frac{1}{r^{\frac{1}{4}}} \sup _{X} t\right) .
$$

Applying it to (6.16), it follows that

$$
\sup _{X} t \leq C r
$$


which is applied back to (6.27) and (6.30) to prove that

$$
\begin{aligned}
& \sup _{X} q \leq C r^{\frac{3}{4}}, \\
& \sup _{X} p \leq C .
\end{aligned}
$$

Step (6). We will derive a good comparison function which bounds $q-p$ from above in order to refine the estimate of $t$.

Lemma 6.7. There exists a constant $\delta>0$ such that if $r$ is sufficiently large, the function $v:=1-|\alpha|^{2}-|\beta|^{2}+\frac{3 \delta}{r}$ obeys the following:

(1) $v \geq 1-|\alpha|^{2}+\frac{2 \delta}{r} \geq \frac{\delta}{r}$.

(2) $\left(\frac{1}{2} \triangle+\frac{r}{4}|\alpha|^{2}\right) v \geq 0$.

The lemma above follows easily from Propositions 5.1 and 5.3.

Lemma 6.8. Define $v_{1}$ by $v_{1}:=v^{1-r^{-\frac{3}{4}}}$. Then it obeys the following:

(1) $2 v \geq v_{1} \geq \frac{1}{2} v$.

(2) $\left(\frac{1}{2} \triangle+\frac{r}{4}|\alpha|^{2}\right) v_{1} \geq \frac{r^{\frac{1}{4}}}{2}|\alpha|^{2}\left(1-|\alpha|^{2}+\frac{2 \delta}{r}\right)$.

Define the function $v_{2}$ by

$$
v_{2}:=\left\{\frac{8}{r^{\frac{1}{4}}}\left(R_{0} \sup _{X} t+\sup _{X} h\right)+4 \sup _{X} q\right\} v_{1} .
$$

The right-hand side of (6.28) is bounded from above by

$$
\begin{aligned}
& \left(R_{0} t+h\right)\left(1-|\alpha|^{2}+\frac{C_{M}}{r^{2}}\right) \\
& \leq\left(R_{0} \sup _{X} t+\sup _{X} h\right)\left(1-|\alpha|^{2}+\frac{C_{M}}{r^{2}}\right),
\end{aligned}
$$

where we have used Proposition 5.3. Thus (2) of Lemma 6.8 implies that, if $r$ is sufficiently large,

$$
\left(\frac{1}{2} \triangle+\frac{r}{4}|\alpha|^{2}\right)\left(q-p-v_{2}\right) \leq 0
$$

on the domain $\Omega_{\frac{1}{2}}\left\{\left.x \in X|| \alpha\right|_{x} ^{2} \geq \frac{1}{2}\right\}$. On the other hand, (1) of Lemma 6.7 and (1) of Lemma 6.8 imply that, if $r$ is sufficiently large,

$$
v_{2} \geq \sup _{X} q \text { on } X \backslash \Omega_{\frac{1}{2}} \text {. }
$$

Therefore, a maximum principle is applied to prove that, if $r$ is sufficiently large,

$$
q-p \leq v_{2} \leq C r^{\frac{3}{4}}\left(1-|\alpha|^{2}\right)+\frac{C}{r^{\frac{1}{4}}} .
$$

Then (6.16), (6.32.2) and Proposition 5.3 imply that

$$
t \leq C r\left(1-|\alpha|^{2}\right)+C \text {. }
$$

Proof Lemma 6.8. The first assertion follows from the inequality

$$
\left(1+\frac{3 \delta}{r}\right)^{\left(r^{-\frac{3}{4}}\right)} \geq \frac{v}{v_{1}} \geq\left(\frac{\delta}{r}\right)^{\left(r^{-\frac{3}{4}}\right)},
$$

where the left-most and right-most sides tend to 1 when $r$ tends to infinity. The second assertion follows from (1) of Lemma 6.7, (1) of Lemma 6.8 and the fact that 
$\triangle\left(f^{b}\right) \geq b(\triangle f) f^{b-1}$ for a smooth positive function $f$ and a constant $b$ that obeys $1 \geq b>0$.

Step (7). We will derive a good comparison function which bounds $p$ from above and verify the required estimate of $t$.

(6.26), (6.38) and (2) of Lemma 6.8 imply that there exists a positive constant $\kappa_{3}$ which depends only on $\left(\omega, J, c_{1}\left(L_{\varrho}\right)\right)$ such that the function $f:=p-\kappa_{3} r^{\frac{3}{4}} v_{1}$ obeys $\left(\frac{1}{2} \triangle+\frac{r}{4}|\alpha|^{2}\right) f \leq 0$. Since $f$ is negative outside some compact set, it follows from a maximum principle that

$$
p \leq \kappa_{3} r^{\frac{3}{4}} v_{1} \leq C r^{\frac{3}{4}}\left(1-|\alpha|^{2}\right)+\frac{C}{r^{\frac{1}{4}}} .
$$

Combining (6.37) with (6.39), it follows that

$$
q \leq C r^{\frac{3}{4}}\left(1-|\alpha|^{2}\right)+\frac{C}{r^{\frac{1}{4}}}
$$

Then (6.16) verifies the required estimate in the statement of Proposition 6.1.

\section{An A PRIORI ESTIMATE FOR THE TOTAL ENERGY INTEGRAL}

Proposition 7.1. There exists a positive constant $C$ which depends only on $\left(\omega, J, c_{1}\left(L_{\varrho}\right)\right)$ and has the following significance: Let $(\alpha, \beta, a)$ be a solution of equations (3.1) with $r \geq 1$. Then it holds that

$$
\left|\nabla_{a} \alpha\right|^{2}+r\left|\tilde{\nabla}_{a} \beta\right|^{2} \leq C\left\{r\left(1-|\alpha|^{2}\right)+1\right\} .
$$

This corresponds to Proposition 2.8 of [T1] and can be proved exactly in the same way by making use of the estimates in Section 5 and Proposition 6.1. See T1] for the proof.

With this in hand, we will devote the latter part of this section to prove

Proposition 7.2. There exists a positive constant $C_{e}$ which depends only on $\left(\omega, J, c_{1}\left(L_{\varrho}\right)\right)$ and has the following significance: Let $(\alpha, \beta, a)$ be a solution of equations (3.1) with $r \geq 1$. Then it holds that

$$
\left.\int_{X} \frac{r}{4}|1-| \alpha\right|^{2} \mid \leq C_{e}
$$

Of course, the pointwise a priori estimates of the integrand that we have obtained in Section 5 do not directly imply the estimate above since a noncompact weakly convex manifold has infinite volume.

Proof. The proof is divided into 3 steps.

Step (1). Let $X_{\frac{1}{2}}$ denote the set $\left\{\left.x \in X|| \alpha\right|_{x} ^{2} \leq \frac{1}{2}\right\}$. We will introduce good subsets $X^{1}, X^{2} \subset X$.

Lemma 7.3. There exists a positive constant $C_{v}$ which depends only on $\left(\omega, J, c_{1}\left(L_{\varrho}\right)\right)$ and has the following significance: Let $V$ be a finite subset $\left\{x_{i}\right\}_{1 \leq i \leq k}$ $\subset X_{\frac{1}{2}}$ such that $B\left(x_{i}, r^{-\frac{1}{2}}\right)$ are mutually disjoint, where $B\left(x_{i}, r^{-\frac{1}{2}}\right)$ denotes the geodesic ball of radius $r^{-\frac{1}{2}}$ with center $x_{i}$. Then $\# V \leq C_{v} r$.

Let $V_{M}$ be one of the sets described in Lemma 7.3 and suppose it is maximal among such sets. Define $X^{1}$ and $X^{2}$ as $\bigcup_{x \in V_{m}} B\left(x_{i}, 2 r^{-\frac{1}{2}}\right)$ and $\bigcup_{x \in V_{m}} B\left(x_{i}, 4 r^{-\frac{1}{2}}\right)$, respectively. Then the following properties hold:

(1) $X_{\frac{1}{2}} \subset X^{1} \subset X^{2}$. 
(2) $\operatorname{dist}\left(X^{1}, X \backslash X^{2}\right) \geq r^{-\frac{1}{2}}$.

(3) $\operatorname{Vol}\left(X^{2}\right) \leq \frac{C}{r}$.

In fact, the first property follows from the maximality of $V_{M}$. The second property is obvious. The third property follows from the bound of $\# V_{M}$ given in Lemma 7.3 .

Proof of Lemma 7.3. Proposition 7.1 and the inequality $\left.|\nabla| \alpha\right|^{2}|\leq 2| \nabla_{a} \alpha|\cdot| \alpha \mid$ imply that there exists a positive constant $C$ such that if $\operatorname{dist}\left(y, X_{\frac{1}{2}}\right) \leq \frac{1}{C} r^{-\frac{1}{2}}$, then $1-|\alpha|_{y}^{2} \geq \frac{1}{4}$. Thus there exists a positive constant $C$ such that

$$
\int_{B\left(x, r^{-\frac{1}{2}}\right)} r\left(1-|\alpha|^{2}\right)^{2} \geq \frac{1}{C} r^{-1}
$$

if $x \in X_{\frac{1}{2}}$. On the other hand, the integral $\int_{X} r\left(1-|\alpha|^{2}\right)^{2}$ is bounded from above by a constant which does not depend on $r$. See Proposition 3.1. Thus we are done.

Step (2). We will introduce a comparison function $\phi$ on $X$ which obeys

$$
\left.|1-| \alpha\right|^{2} \mid \leq \phi \text { on } X \backslash X^{2} .
$$

Let $C_{g}$ be a constant determined later such that $0<C_{g} \leq 1$ and such that it depends only on the weakly convex almost Kähler structure.

Lemma 7.4. There exist positive constants $C_{c}$ and $\epsilon_{c}$ which depend only on $\left(\omega, J, c_{1}\left(L_{\varrho}\right)\right)$ and have the following significance: Let $y \in X \backslash X^{2}$.

(1) If $\operatorname{dist}\left(y, X^{1}\right) \geq C_{g} \sigma(y)$, then

$$
\left.|1-| \alpha\right|_{y} ^{2} \mid \leq C_{c} \exp ^{-\epsilon_{c} \sqrt{r}\left\{C_{g} \sigma(y)\right\}} .
$$

(2) If $\operatorname{dist}\left(y, X^{1}\right) \leq C_{g} \sigma(y)$, then

$$
\left.|1-| \alpha\right|_{y} ^{2} \mid \leq C_{c} \max _{x_{i} \in V_{M}}\left\{\exp ^{-\epsilon_{c} \sqrt{r}\left\{\operatorname{dist}\left(y, x_{i}\right)-2 r^{-\frac{1}{2}}\right\}}\right\} .
$$

Proof of Lemma 7.4. Define $d_{y}$ by

$$
d_{y}:=\min \left\{C_{g} \sigma(y), \min _{x_{i} \in V_{M}} \operatorname{dist}\left(y, x_{i}\right)-2 r^{-\frac{1}{2}}\right\} .
$$

Then we see that $|\alpha|^{2} \geq \frac{1}{2}$ on the geodesic ball $B\left(y, d_{y}\right)$. Let $\tilde{e}_{y}: T X_{y} \mapsto X$ be the map defined by $\tilde{e}_{y}(v):=\exp _{y}\left(d_{y} v\right)$. Then the pull-back $(\underline{\alpha}, \underline{\beta}, \underline{a}):=\tilde{e}_{y}^{*}(\alpha, \beta, a)$ is a solution of (3.1) with rescaling parameter $r d_{y}^{2}$. The pull-back Riemannian metric and symplectic form are a priori bounded in the sense of Definition 1.1. Then the assertion is an immediate consequence of the following:

Proposition 7.5 ([K-M2]). Let $(\alpha, \beta, a)$ be a solution of equations (3.1) defined on the unit ball $B_{1}$ with rescaling parameter $r_{1}>0$ and suppose that $\eta$ obeys $\|\eta\|_{C^{l}\left(B_{1}\right)} \leq e^{-\delta_{1} r_{1}}$ for a positive constant $\delta_{1}$. Then there exist positive constants $C_{u}$ and $\epsilon_{1}$ that depend only on $\delta_{1}$ and on the Riemannian metric and the symplectic form of the unit ball and have the following significance: If $|\alpha|^{2} \geq \frac{1}{2}$ on $B_{1}$, then

$$
\sup _{B_{\frac{1}{2}}}\left\{\left.\left.|1-| \alpha\right|^{2}|+| \beta\right|^{2}+\left|\nabla_{a} \alpha\right|^{2}+\left|\tilde{\nabla}_{a} \beta\right|^{2}+\left|F_{a}\right|\right\} \leq C_{u} \exp ^{-\epsilon_{1} \sqrt{r_{1}}} .
$$


See Proposition 3.22 in $\bar{K}-\mathrm{M} 2$ for the proof.

We may define the comparison function $\phi$ by

$$
\phi(y):=C_{c}\left\{\sum_{x_{i} \in V_{M}} \Omega_{B\left(x_{i}, \sigma\left(x_{i}\right)\right)}(y) \cdot \exp ^{-\epsilon_{c} \sqrt{r}\left\{\operatorname{dist}\left(y, x_{i}\right)-2 r^{-\frac{1}{2}}\right\}}+\exp ^{-\epsilon_{c} \sqrt{r}\left\{C_{g} \sigma(y)\right\}}\right\},
$$

where the symbol $\Omega_{A}$ for $A \subset X$ denotes the characteristic function of $A$. Then Lemma 7.4 and the following lemma verify (7.4).

Lemma 7.6. There exists a constant $C_{g}$ such that $0<C_{g} \leq 1$ and such that it depends only on $(\omega, J, \sigma)$ and has the following significance: If $r$ is sufficiently large and if $\operatorname{dist}(y, x) \leq C_{g} \sigma(y)+2 r^{-\frac{1}{2}}$, then $\operatorname{dist}(y, x) \leq \sigma(x)$.

The lemma above follows from (4) of Property (A) in Definition 1.1.

Step (3). We will verify the required estimate for the energy integral.

We will estimate first the integral over $X \backslash X^{2}$. It follows from (7.4) that

$$
\begin{aligned}
\int_{X \backslash X^{2}} & \left.|1-| \alpha\right|^{2} \mid \leq \int_{X} \phi \\
\leq & C_{c} \sum_{x_{i} \in V_{M}} \int_{B\left(x_{i}, \sigma\left(x_{i}\right)\right)} \exp ^{-\epsilon_{c} \sqrt{r}\left\{\operatorname{dist}\left(y, x_{i}\right)-2 r^{-\frac{1}{2}}\right\}} \\
& +C_{c} \int_{X} \exp ^{-\epsilon_{c} \sqrt{r}\left\{C_{g} \sigma(y)\right\}} .
\end{aligned}
$$

Lemma 7.3 implies that the first term of the right-hand side of (7.8) is bounded from above by

$$
C_{c}\left(C_{v} r\right) C_{l} \int_{\mathbb{R}^{4}} \exp ^{-\epsilon_{c} \sqrt{r}\left(|y|-2 r^{-\frac{1}{2}}\right)} .
$$

Here $C_{l}$ is a positive constant which depends only on $(\omega, J)$ and has the following significance: Let $x \in X$. Fix an isometry $P_{x}: \mathbb{R}^{4} \mapsto T X_{x}$ and define the Riemannian metric $g_{x}$ on $B(0, \sigma(x)) \subset \mathbb{R}^{4}$ by $g_{x}:=\left(\exp _{x} \circ P_{x}\right)^{*}\left(g_{J}\right)$ where $g_{J}$ is the Riemannian metric of $X$. Then it follows that $\int_{\partial B(0, R)} i_{\frac{\partial}{\partial R}} d \operatorname{vol}_{g_{i}} \leq 4 \pi^{2} C_{l} R^{3}$ where the coordinate $R$ stands for the distance from the origin. The existance of $C_{l}$ is assured by the weak convexity of $X$.

The integrand in (7.9) is no more than $\exp ^{2 \epsilon_{c}} \cdot \exp ^{-\epsilon_{c} \sqrt{r}|y|}$. Thus (7.9) is no more than

$$
\begin{aligned}
C r \int_{0}^{\infty} & d R R^{3} \exp ^{-\epsilon_{c} \sqrt{r} R} \\
& =\frac{C}{r} \int_{0}^{\infty} d Q Q^{3} \exp ^{-\epsilon_{c} Q} \\
& \leq \frac{C}{r} .
\end{aligned}
$$

The second term of the right-hand side of (7.8) is estimated as follows (see Property (B) of the Definition 1.1):

$$
\begin{aligned}
C_{c} \int_{\mathbb{R}} d y g_{\sigma} \exp ^{-\epsilon_{c} \sqrt{r}\left\{C_{g} y\right\}} & \leq C \int_{\mathbb{R}} d y y^{\max \left(1, \epsilon_{0}\right)} \exp ^{-\epsilon_{c} \sqrt{r}\left\{C_{g} y\right\}} \\
& \leq C r^{-\max \left(1, \frac{1+\epsilon_{0}}{2}\right)} .
\end{aligned}
$$


Thus we have obtained

$$
\left.\int_{X \backslash X^{2}}|1-| \alpha\right|^{2} \mid \leq C r^{-1}
$$

On the other hand, the third property of $X^{2}$ and Proposition 5.2 imply that

$$
\left.\int_{X^{2}}|1-| \alpha\right|^{2} \mid \leq C \cdot \operatorname{Vol}\left(X^{2}\right) \leq C r^{-1} .
$$

Therefore, the required estimate (7.2) is verified.

\section{A MONOTONICITY FORMULA FOR LOCAL ENERGY INTEGRALS}

We will prove in this section a monotonicity formula for local energy integral, the formula which is a slightly refined version of Proposition 3.2 in [T1.

Proposition 8.1. Let $(\alpha, \beta, a)$ be a solution of equations (3.1) with $r \geq 1$. For $x \in X$ define the function $\mathcal{E}_{x}$ by $\mathcal{E}_{x}(R):=\left.\int_{B(x, R)^{\frac{r}{4}}}|1-| \alpha\right|^{2} \mid$. There exist positive constants $\mu_{7}, \mu_{8}, \mu_{9}$ and $\rho_{0}$ with $1 \geq \rho_{0}>0$ which depend only on $\left(\omega, J, c_{1}\left(L_{\varrho}\right)\right)$ and have the following significance:

If $\rho_{0} \sigma(x) \geq R \geq 0$, then

$$
\mathcal{E}_{x}(R) \leq \frac{R}{2}\left(1+\mu_{7} \frac{R}{\sigma(x)}\right)\left(1+\frac{\mu_{8}}{r^{\frac{1}{4}}}\right) \frac{d}{d R} \mathcal{E}_{x}(R)+\frac{\mu_{9}}{r^{\frac{1}{4}}} R^{4} .
$$

We omit the proof since it is exactly the same as that of Proposition 3.2 in [T1]. But it is essential to make use of Proposition 6.1. Our formula is different from the one in [T1] in that it has $r^{-\frac{1}{4}}$ factor in the second term of the right-hand side, which is due to the existence of the $r^{-\frac{1}{4}}$ factor in the second term of the right-hand side of (6.1) (and that of the $r^{-1}$ factor in the second term of the R.H.S. of (5.7)).

By making use of it, we will prove

Proposition 8.2. There exist a positive constant $C_{f}$ and a positive function $\pi_{0}$ which depend only on $\left(\omega, J, C_{1}\left(L_{\varrho}\right)\right)$ and have the following significance: If $|\alpha|_{x}^{2} \leq \frac{1}{2}$ and if $R$ obeys $r \geq \pi_{0}(R)$ and $\rho_{0} \sigma(x) \geq R$, then

$$
\mathcal{E}_{x}(R) \geq \frac{1}{C_{f}} R^{2}
$$

Proof of Proposition 8.2. We will mimic the proof of Proposition 3.1 in [T1].

Define the function $f_{x}$ by

$$
f_{x}(R):=-2\left(1+\frac{\mu_{8}}{r^{\frac{1}{4}}}\right)^{-1} \log \left(\frac{R}{1+\mu_{7} \frac{R}{\sigma(x)}}\right) .
$$

It follows from (8.1) that if $\rho_{0} \sigma(x) \geq R \geq 0$, then

$$
\frac{d}{d R}\left(\exp ^{f} \mathcal{E}_{x}\right) \geq-C r^{-\frac{1}{4}} R^{3} \exp ^{f}
$$

Fix a positive constant $R_{0}$. Let $x \in X$ satisfy the condition that $\rho_{0} \sigma(x) \geq R_{0}$. There exists a positive constant $C_{R_{0}}$ which depends only on $R_{0}$ and on $\left(\omega, J, c_{1}\left(L_{\varrho}\right)\right)$ and has the following significance: If $r^{-\frac{1}{2}} \leq R \leq R_{0}$, then $\exp ^{f(R)} \leq C_{R_{0}} R^{-2}$. Here we have used the fact that $\lim _{r \rightarrow \infty} r^{\left(r^{-\frac{1}{4}}\right)}=0$. This implies that if $r^{-\frac{1}{2}} \leq R \leq R_{0}$, then

$$
\frac{d}{d R}\left(\exp ^{f} \mathcal{E}_{x}\right) \geq-C \cdot C_{R_{0}} r^{-\frac{1}{4}} R
$$


Integrating (8.4) over $\left[r^{-\frac{1}{2}}, R_{0}\right]$, we obtain

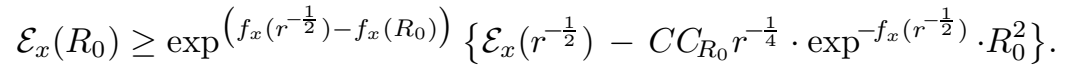

We will estimate the right-hand side of (8.5);

Lemma 8.3. If $|\alpha|_{x}^{2} \leq \frac{1}{2}$, then $\mathcal{E}_{x}\left(r^{-\frac{1}{2}}\right) \geq \zeta_{0} r^{-1}$ for some positive constant $\zeta_{0}$ that depends only on $\left(\omega, J, c_{1}(L \varrho)\right)$.

On the other hand, we can easily check that

$$
\begin{aligned}
& \lim _{r \rightarrow \infty} r^{-1}\left(\exp \left(f_{x}\left(r^{-\frac{1}{2}}\right)-f_{x}\left(R_{0}\right)\right)\right)=\left(1+\mu_{7} \frac{R_{0}}{\sigma(x)}\right)^{-2} R_{0}^{2}, \\
& \lim _{r \rightarrow \infty} r\left(C_{R_{0}} r^{-\frac{1}{4}} \cdot \exp ^{-f_{x}\left(r^{-\frac{1}{2}}\right)} \cdot R_{0}^{2}\right)=0 .
\end{aligned}
$$

Therefore, if $r$ is no less than a sufficiently large constant $\pi_{0}\left(R_{0}\right)$ that depends only on $R_{0}$, we see that

$$
\mathcal{E}_{x}\left(R_{0}\right) \geq \frac{1}{2}\left(1+\mu_{7} \frac{R_{0}}{\sigma(x)}\right)^{-2} \zeta_{0} R_{0}^{2} .
$$

The assumption that $\rho_{0} \sigma(x) \geq R_{0}$ implies that the coefficient of $\zeta_{0} R_{0}^{2}$ in the righthand side is no less than a positive constant that depends only on $\left(\omega, J, c_{1}\left(L_{\varrho}\right)\right)$. Thus we are done.

Proof of Lemma 8.3. Proposition 7.1 implies that there exists a positive constant $C$ such that $1-|\alpha|^{2} \geq \frac{1}{4}$ on the geodesic ball $B\left(x, \frac{1}{C} r^{-\frac{1}{2}}\right)$ if $|\alpha|_{x}^{2} \leq \frac{1}{2}$. Thus it follows that

$$
\mathcal{E}_{x}\left(r^{\frac{1}{2}}\right)=\left.\frac{r}{4} \int_{B\left(x, r^{\frac{1}{2}}\right)}|1-| \alpha\right|^{2} \mid \geq \frac{r}{16} \operatorname{Vol}\left\{B\left(x, r^{-\frac{1}{2}} \cdot \min \left(1, C^{-1}\right)\right)\right\} \geq \frac{1}{C r} .
$$

\section{Final arguments for Main Theorem}

Recall that $C_{e}, \rho_{0}$ and $C_{f}$ are the constants defined in Proposition 7.2, 8.1 and 8.2 respectively and that $\pi_{0}$ is the function defined in Proposition 8.2.

Proposition 9.1. There exists a constant $R_{M}$ which depends only on $\left(\omega, J, c_{1}\left(L_{\varrho}\right)\right)$ and has the following significance: Let $(\alpha, \beta, a)$ be a solution of (3.1). If $x \in X$ obeys $|\alpha|_{x}^{2} \leq \frac{1}{2}$ and if $r \geq \pi_{0}\left(R_{M}\right)$, then $\rho_{0} \sigma(x)<R_{M}$.

Proof. We may take $R_{M}$ to be $\sqrt{2 C_{e} C_{f}}$. In fact, suppose that $|\alpha|_{x}^{2} \leq \frac{1}{2}, r \geq$ $\pi_{0}\left(R_{M}\right)$ and $\rho_{0} \sigma(x) \geq R_{M}$. Then Proposition 8.2 implies that

$$
\mathcal{E}_{x}\left(R_{M}\right) \geq \frac{1}{C_{f}} R_{M}^{2}=2 C_{e},
$$

which contradicts the assertion of Proposition 7.2 that $C_{e} \geq \mathcal{E}_{x}\left(R_{M}\right)$.

Corollary 9.2. There exist a compact set $K_{M} \subset X$ and a positive constant $C$ which depend only on $\left(\omega, J, c_{1}\left(L_{\varrho}\right)\right)$ and have the following significance: Any solution $(\alpha, \beta, a)$ of (3.1) with $r \geq 1$ obeys $|\alpha|^{2} \geq \frac{1}{2}$ on $X \backslash K_{M}$.

Combining Corollary 9.2 with Proposition 7.5, we immediately obtain 
Proposition 9.3. There exist positive constants $\epsilon_{2}$ and $C_{d}$ which depend only on $\left(\omega, J, c_{1}\left(L_{\varrho}\right)\right)$ and have the following significance: Let $(\alpha, \beta, a)$ be a solution of (3.1) with $r \geq 1$. Then it obeys

$$
\left.\left.|1-| \alpha\right|^{2}|+| \beta\right|^{2}+\left|\nabla_{a} \alpha\right|^{2}+\left|\tilde{\nabla}_{a} \beta\right|^{2}+\left|F_{a}\right| \leq C_{d} \exp ^{-\epsilon_{2} \sqrt{r} \sigma} \text { on } X \backslash K_{M} .
$$

Once Proposition 9.3 is achieved, the arguments in [T1] can be applied to our case almost directly to prove Theorem 4.1. But we need the following two minor modifications to complete the proof:

The first one is in Lemma 3.5 of [T1]. Let $\Omega_{M}$ be the domain $\{x \in X \mid \sigma(x)<$ $\left.2 \max _{K_{M}} \sigma\right\}$. Then $\bar{\Omega}_{M}$ is compact. We may assume that $\partial \Omega_{M}$ is smooth. With this understood, we require the function $u$ in the statement not to be an element of $C^{\infty}(X)$ but that of $C^{\infty}\left(\Omega_{M}\right) \cap C^{0}\left(\overline{\Omega_{M}}\right)$ with $\left.u\right|_{\partial \Omega_{M}} \equiv 0$. Accordingly, we replace the Green function $G$ in the proof by the fundamental solution of the Dirichlet problem of $d^{*} d$ with domain $\Omega_{M}$. Then by making use of the modified $u$ with Proposition 9.4, we can prove exactly the same result as in (d) of Section 3 in [T1].

The second modification is in Part (1) of the proof of Lemma 4.3 in T1, where we must bound the function $\left|P^{+} F_{a}\right|^{2}-\left|P^{-} F_{a}\right|^{2}$ from below by a function $f$ that obeys $\|f\|_{L^{1}(X)} \leq C$ for a constant $C$ which depends only on $\left(\omega, J, c_{1}\left(L_{\varrho}\right)\right)$. For this purpose, we may define $f$ by

$$
f:=- \begin{cases}\kappa\left\{\left.r|1-| \alpha\right|^{2} \mid+1\right\} & \text { on } \Omega_{M}, \\ \kappa C_{d}^{2} \exp ^{-2 \epsilon_{2} \sqrt{r} \sigma} & \text { on } X \backslash \Omega_{M},\end{cases}
$$

where the constant $\kappa$ is chosen sufficiently large.

\section{An Application}

Let $\Gamma$ be a discrete subgroup of $\mathrm{SU}(2)$. The classification of such groups is well known. They are in one-to-one correspondence with the Dynkin diagrams of type $A_{n}, D_{n}$ and $E_{6}, E_{7}, E_{8}$.

Let $Y_{1}, Y_{2}$ and $Y_{3}$ be the standard basis of the Lie algebra $\mathfrak{s u}(2)$ which we regard as right invariant vector fields. Define the contact 2-plane field $\zeta$ to be the span of $Y_{1}$ and $Y_{2}$, which is called the standard contact structure of the 3 -sphere. $\zeta$ drops to the quotient space $M_{\Gamma}:=\mathrm{SU}(2) / \Gamma$ as a contact structure denoted by $\zeta_{\Gamma}$.

Theorem 10.1. Let $\left(X_{0}, \omega\right)$ be a symplectic filling of $\left(M_{\Gamma}, \zeta_{\Gamma}\right)$ such that it is minimal.

(1) The intersection form of $X_{0}$ is negative definite.

(2) The trivialization of the canonical line bundle $K_{X_{0}}$ given over $\partial X_{0}$ by $Y_{1}$ extends to the interior of $X_{0}$. In particular, $X_{0}$ must be a spin manifold.

Notice that, if we regard $\zeta_{\Gamma}$ as a complex line bundle, it is canonically isomorphic to $\left.K_{X_{0}}\right|_{\partial X_{0}}$.

Remark 10.2. (1) Ohta and Ono $\mathrm{O}-\mathrm{O}$. proved this theorem in the case where the Dynkin diagram of $\Gamma$ is $E_{8}$, that is, $M_{\Gamma}$ is the Poincare homology of the 3-sphere.

(2) Combining our result with that in $\left[\mathrm{F}\right.$, we get a good estimate of $b_{2}\left(X_{0}\right)$. In particular, if $\Gamma$ corresponds to $E_{8}$, the intersection matrix of $X_{0}$ must be $-E_{8}$.

Proof of (2) of Theorem 10.1. Denote by $Y_{1}^{*}, Y_{2}^{*}, Y_{3}^{*}$ the standard dual basis of $\mathfrak{s u}(2)^{*}$ which we regard as right-invariant 1-forms of SU(2). Proposition 1.3 implies that there exists a weakly convex almost Kähler manifold $(X, \tilde{\omega}, J)$ obeying: 
(1) $X \cong X_{0} \cup_{i d} M_{\Gamma} \times[1, \infty)$ as smooth manifolds.

(2) $\left.\tilde{\omega}\right|_{X_{0}}=\omega$.

(3) There exists a positive constant $l \geq 1$ such that $\left.\tilde{\omega}\right|_{M_{\Gamma} \times[l, \infty)}=d\left(t^{2} Y_{3}^{*}\right)$ and such that $\left.J\right|_{M_{\Gamma} \times[l, \infty)}$ obeys the formulae $J\left(Y_{1}\right)=Y_{2}, J\left(Y_{2}\right)=-Y_{1}, J\left(\partial_{t}\right)=$ $Y_{3}, J\left(Y_{3}\right)=-\partial_{t}$.

For simplicity, we may assume that $l=1$.

All through the later arguments, we regard $X_{0}$ as a subset of $X$ and denote by $X^{+}$the conical end $M_{\Gamma} \times[1, \infty)$.

Define the 1-parameter family of symplectic forms $\left\{\omega_{\nu}\right\}_{0 \leq \nu \leq 1}$ on $X^{+}$by

$$
\omega_{\nu}:=d\left\{t^{2}\left((\cos \pi \nu) Y_{3}^{*}+(\sin \pi \nu) Y_{1}^{*}\right)\right\}
$$

These $\omega_{\nu}$ are self-dual 2 -forms of length $\sqrt{2}$ with respect to $\left.g_{J}\right|_{X^{+}}$. Hence, for each $\nu$ there exists a unique almost complex structure $J_{\nu}$ compatible with $\omega_{\nu}$ such that the associated metric $\omega_{\nu}(*, J *)$ coincides with $\left.g_{J}\right|_{X^{+}}$. Then we see that $\left.(\tilde{\omega}, J)\right|_{X^{+}}=$ $\left(\omega_{0}, J_{0}\right)=\left(-\omega_{1},-J_{1}\right)$.

Remark. These $J_{\nu}$ are integrable. In fact, $\left.g_{J}\right|_{X^{+}}$is a hyper-Kähler metric.

For the time being, we fix an element $(s, \varrho) \in S(X, \omega)$.

Let $\mathbb{I}$ be the unit length section of $\left.W_{s}\right|_{X^{+}}$given as the pull-back of $(1,0) \in$ $\Gamma\left(W_{s_{\tilde{\omega}}}\right) \equiv \Gamma(\mathbb{C} \oplus K)$ through the identification map $\varrho:\left.\left.W_{s}\right|_{X^{+}} \mapsto W_{s_{\tilde{\omega}}}\right|_{X^{+}}$. Notice that $\rho(\tilde{\omega}) \mathbb{I}=-2 \sqrt{-1} \mathbb{I}$. We see that there exists a smooth 1-parameter family of unit length sections $\left\{\mathbb{I}_{\nu}\right\}_{0 \leq \nu \leq 1}$ which obeys the equation $\rho\left(\omega_{\nu}\right) \mathbb{I}_{\nu}=-2 \sqrt{-1} \mathbb{I}_{\nu}$ and the initial condition $\mathbb{I}_{0}=\mathbb{I}$. This induces a smooth 1-parameter family of the isomorphisns $\varrho_{\mathbb{I}_{\nu}}:\left.\left.W_{s}\right|_{X^{+}} \mapsto W_{s_{\tilde{\omega}}}\right|_{X^{+}}$by imposing the condition $\varrho_{\mathbb{I}_{\nu}}\left(\mathbb{I}_{\nu}\right)=(1,0)$. Notice that $\varrho_{\mathbb{I}_{0}}=\varrho$. We can easily show that the family $\left\{\left(\omega_{\nu}, J_{\nu}, \varrho_{\mathbb{I}_{\nu}}\right)\right\}_{0 \leq \nu \leq 1}$ satisfies the assumption in the statement of the first property of the monopole invariant $S W$ (see Section 1). Thus it follows that

$$
S W(X, \tilde{\omega}, J, s, \varrho)= \pm S W\left(X,-\tilde{\omega},-J, s, \varrho_{\mathbb{I}_{1}}\right) .
$$

Let $-s$ be the $\operatorname{Spin}^{c}$ structure obtained from $s$ by changing the sign of the complex structure. Then $W_{-s}$ is canonically isomorphic to $W_{s}$ as a real vector bundle. Denote by $\overline{\mathbb{I}}_{1}$ the section of $W_{-s}$ that corresponds to $\mathbb{I}_{1}$. Then $\rho(\tilde{\omega}) \overline{\mathbb{I}_{1}}=$ $-2 \sqrt{-1} \overline{\mathbb{I}}_{1}$ since $\rho(\tilde{\omega}) \mathbb{I}_{1}=2 \sqrt{-1} \mathbb{I}_{1}$. Since the change of the sign of the complex structure does not affect the underlying equation, we obtain

$$
S W\left(X,-\tilde{\omega},-J, s, \varrho_{\mathbb{I}_{1}}\right)= \pm S W\left(X, \tilde{\omega}, J,-s, \varrho_{\mathbb{I}_{1}}\right) .
$$

Now suppose that the element $(s, \varrho)$ that we have fixed so far to be $\left(s_{\tilde{\omega}}, \mathrm{id}\right)$. Since $S W\left(X, \tilde{\omega}, J, s_{\tilde{\omega}}, \mathrm{id}\right)=1$ (see Section 1), by combining the two formulae above, we have

$$
S W\left(X, \tilde{\omega}, J,-s_{\tilde{\omega}}, \varrho_{\overline{\mathbb{I}_{1}}}\right)= \pm 1 .
$$

The corresponding line bundle to $-s_{\tilde{\omega}}$ is $K$ since $W_{-s_{\tilde{\omega}}}=K \oplus \mathbb{C}=(\mathbb{C} \oplus \bar{K}) \otimes K$. Hence, we have only to show that $\left(K, \varrho_{\mathbb{I}_{1}}\right)$ coincides with $(\mathbb{C}$, id $)$.

Assume to the contrary. Then applying Theorem 4.1, we obtain a non-empty, compact $J$-holomorphic curve $D \subset X$ such that P.D. $[D]=c_{1}\left(K, \varrho_{\overline{\mathbb{I}}_{1}}\right) \in \mathrm{H}_{\mathrm{cpt}}^{2}(X, \mathbb{Z})$. Taking multiplicities into account, $D$ is written as

$$
D=\sum_{i=1}^{k} n_{i} D_{i}
$$


where the $D_{i}$ 's are mutually distinct and non-multiple such that each $D_{i}$ is the image of a non-constant $J$-holomorphic map from a connected compact Riemann surface. $n_{i}$ is a positive integer that represents the multiplicity of $D_{i}$ in $D$. The minimality of $\left(X_{0}, \omega\right)$ means that $(X, \tilde{\omega}, J)$ contains no embedded $J$-holomorphic rational curve whose self-intersection number is -1 . Thus the argument in the proof of Proposition 7.1 in [T1] shows that, if $J$ is generic, each $D_{i}$ is a smooth submanifold, $D_{i} \cap D_{j}=\emptyset$ for $i \neq j$ and $D_{i} \cdot D_{i} \geq 0$. Since $\partial X_{0}$ is a rational homology 3 -sphere, the intersection form of $X$ is non-degenerate. Further, $\left[D_{i}\right] \in \mathrm{H}_{2}(X, \mathbb{Z})$ is not zero since $\int_{D_{i}} \tilde{\omega}>0$. Thus it follows that $b_{2}^{+}\left(X_{0}\right) \geq 1$, which contradicts assertion (1).

Remark 10.3. In general, a compact $J$-holomorphic curve $D$ in a weakly convex almost Kähler manifold $(X, \omega, J)$ is contained in a compact set $K_{[D]}$ which is detemined a priori by the value $\langle[D]$, $[\omega]\rangle$ due to the monotonicity formula of energy density. Thus to have $J$ generic, it is sufficient to consider the space $\mathfrak{J}$ of compatible complex structures which agree with a fixed almost complex structure outside a fixed compact set. In fact, we can show that there exists a Baire subset of $\mathfrak{J}$ whose elements have the needed genericity for all $J$-holomorphic curves of a fixed homology class.

Proof of (1) of Theorem 10.1. This follows from the standard necking argument in gauge theory, which is well-known by the experts. Hence, we will give here only the sketch of the proof. See [M-S-T] ) and $[\mathrm{F}]$ for the details.

We will derive a contradiction by assuming that $b_{2}^{+}\left(X_{0}\right)>0$.

Let $X$ and $X^{+}$be as defined in the proof of assertion (1). Perturb the Riemannian metric of $X$ only near $\partial X_{0}\left(=-\partial X^{+}\right)$so that some regular neighborhood of $\partial X_{0}$ is isometric to the Cartesian product of $\left(M_{\Gamma}, g_{M}\right)$ with a small open interval and so that $\partial X_{0}$ is totally geodesic. Here $g_{M}$ is the standard Riemannian metric of $M_{\Gamma}$. Splitting $X$ along $\partial X_{0}$ into pieces and gluing back $\left(X_{R}^{m}, g_{R}^{m}\right):=$ $\left(M_{\Gamma} \times[-R, R], g_{M}+d t^{2}\right)$ with $R>1$ between them, we obtain the new Riemannian manifold $\left(X_{R}, g_{R}\right)$ with no boundary.

Fix an element $(s, \varrho) \in S(X, \tilde{\omega})$ and consider the following monopole equation on $X_{R}$ with variables $(\Phi, B) \in \Gamma\left(W_{s}^{+}\right) \times \mathcal{A}\left(\operatorname{det} W_{s}^{+}\right)$:

$$
\begin{aligned}
\mathcal{D}_{a} \Phi & =0, \\
F_{a}^{+} & =\rho^{-1}\left(\Phi \Phi^{*}\right)_{0}-\frac{\sqrt{-1}}{2} \tau^{+} \cdot \tilde{\omega}+\tau_{m} \cdot \operatorname{Pr}^{+}\left\{\pi^{*}\left(\mu_{m}\right)\right\}+\mu_{1}+\mu_{2} .
\end{aligned}
$$

We will explain the notation in order:

1. $\rho^{-1}: \sqrt{-1} \operatorname{SkewEnd}\left(W_{s}^{+}\right) \mapsto \Lambda^{+}$is the inverse of the bundle isomomorphism $\rho$.

2. $\tau^{+} \in C^{\infty}\left(X_{R}\right), \operatorname{supp}\left(\tau^{+}\right) \subset X^{+}$and $\tau_{+} \equiv 1$ outside some compact set.

3. $\tau_{m} \in C^{\infty}\left(X_{R}\right), \operatorname{supp}\left(\tau_{m}\right) \subset X_{R}^{m}$ and $\tau_{m} \equiv 1$ on $M_{\Gamma} \times[-R+1, R-1] \subset X_{R}^{m}$.

4. $\mu_{m}$ is an exact 2-form of $M_{\Gamma}$.

5. $\pi: M_{\Gamma} \times[-R, R] \mapsto M_{\Gamma}$ is the natural projection.

6. $\mu_{1}$ is a self-dual 2 -form such that $\operatorname{supp}\left(\mu_{1}\right) \subset X_{0}$.

7. $\mu_{2}$ is a self-dual 2-form such that $\operatorname{supp}\left(\mu_{2}\right) \subset X^{+}$and such that $\left\|\mu_{2}\right\|_{\mathcal{N}\left(X^{+}\right)}<$ $\infty$

Notice that this equation can be written in the form of (3.1) with $r=1$ when restricted to the complement of a sufficiently large compact set. We impose the 
same boundary condition as in Section 3 to $(\Phi, B)$ and define the moduli space $\mathcal{M}_{R}$ to be the gauge equivalence classes of the solutions.

Assertion 10.4. For the generic choice $\left(\mu_{m}, \mu_{1}, \mu_{2}\right)$ (which can be taken smooth), the equation is transverse, that is, the linearized equation at any solution is surjective.

The important feature of this equation is that it restricts to $X_{R}^{m} \cong M_{\Gamma} \times$ $[-R, R]$ as the gradient flow equation of the perturbed Chern-Simons-Dirac functional C.S.D $\mu_{m}$ when adopting temporal gauge. In general, a stationary point of C.S.D $\mu_{m}$ is a solution of the following, reduced-to-3-dimensional monopole equation:

$$
\begin{aligned}
\mathcal{D}_{B} \Phi & =0, \\
\left.\rho\left(F_{B}\right)\right|_{W_{s^{\prime}}^{+}} & =\left(\Phi \Phi^{*}\right)_{0}+\mu_{m} .
\end{aligned}
$$

Here the $\operatorname{Spin}^{c}$ structure $s^{\prime}$ on $M_{\Gamma}$ is such that $\left.\pi^{*}\left(s^{\prime}\right) \cong s\right|_{X_{R}^{m}}$. In general, the same argument that derives the a priori $C^{0}$ estimate of monopole equations shows that if $\left\|\mu_{m}\right\|_{C^{0}}$ is sufficiently small and if the Riemannian metric has positive scalar curvature, there are only reducible solutions, that is, $\Phi \equiv 0$. Thus we have

Lemma 10.5. The stationary point of C.S. $D_{\mu_{m}}$ on $M_{\Gamma}$ is reducible and unique up to gauge equivalence if $\mu_{m}$ is sufficiently small. Further, for generic $\mu_{m}$ the Hessian of C.S.D $\mu_{\mu_{m}}$ at the stationary point $\left(0, B_{0}\right)$ is non-degenerate when restricted to the orthogonal complement of the tangent space of the gauge orbit containing $\left(0, B_{0}\right)$.

Gluing the half cylinder $\left(M_{\Gamma} \times[0, \infty), g_{M}+d t^{2}\right)$ along the boundary to $X_{0}$ and $X^{+}$respectively, we get two Riemannian manifolds with no boundary denoted by $X_{1}$ and $X_{2}$ respectively. Assume that $\mathcal{M}_{R}$ be non-empty for all sufficientlly large $R$. Then taking $R$ to infinity and following the standard necking argument (see M-S-T] , we obtain on each $X_{i}$ a solution $\left(\Phi_{i}, B_{i}\right)$ described as follows:

(1) $\left(\Phi_{1}, B_{1}\right) \in \Gamma\left(\left.W_{s}^{+}\right|_{X_{1}}\right) \times \mathcal{A}\left(\left.\operatorname{det} W_{s}^{+}\right|_{X_{1}}\right)$ obeys

$$
\begin{aligned}
& \mathcal{D}_{B_{1}} \Phi_{1}=0, \\
& F_{B_{1}}^{+}=\rho^{-1}\left(\Phi \Phi^{*}\right)_{0}+\mu_{1}+\tau \cdot \operatorname{Pr}^{+}\left\{\pi^{*}\left(\mu_{m}\right)\right\}, \\
& \left\|\left.\left(\Phi_{1}, B_{1}\right)\right|_{M_{\Gamma} \times[0, \infty)}-\pi^{*}\left(0, B_{0}\right)\right\|_{W^{k, 2}\left(M_{\Gamma} \times[0, \infty)\right)}<\infty .
\end{aligned}
$$

(2) $\left(\Phi_{2}, B_{2}\right) \in \Gamma\left(\left.W_{s}^{+}\right|_{X_{2}}\right) \times \mathcal{A}\left(\left.\operatorname{det} W_{s}^{+}\right|_{X_{2}}\right)$ obeys

$$
\begin{aligned}
& \mathcal{D}_{B_{2}} \Phi_{2}=0, \\
& F_{B_{2}}^{+}=\rho^{-1}\left(\Phi \Phi^{*}\right)_{0}-\frac{\sqrt{-1}}{2} \tau^{+} \cdot \tilde{\omega}+\mu_{2}+\tau \cdot \operatorname{Pr}^{+}\left\{\pi^{*}\left(\mu_{m}\right)\right\}, \\
& \left\|\left.\left(\Phi_{2}, B_{2}\right)\right|_{M_{\Gamma} \times[0, \infty)}-\pi^{*}\left(0, B_{0}\right)\right\|_{W^{k, 2}\left(M_{\Gamma} \times[0, \infty)\right)}<\infty, \\
& \left\|\left.\left(\Phi_{2}, B_{2}\right)\right|_{X^{+}}-\left(\mathbb{I}, B_{d}\right)\right\|_{W^{k, 2}\left(X^{+}\right)}<\infty .
\end{aligned}
$$

Here $k$ is fixed sufficiently large and $\tau \in C_{0}^{\infty}\left(M_{\Gamma} \times[0, \infty)\right)$ such that $\tau \equiv 1$ on $M_{\Gamma} \times[1, \infty) . \quad B_{d}$ in (2) is the connection of $\operatorname{det}\left(W_{s_{\tilde{\omega}}}\right)$ that corresponds to the trivial connection of the trivial line bundle. (See Section 2.)

Now we have to define suitable moduli spaces $\mathcal{M}_{i}$ in which the gauge equivalence classes of the solutions $\left(\Phi_{i}, B_{i}\right)$ should live respectively. The usual Sobolev norm is not suitable for this purpose since the "boundary value" $\left(0, B_{0}\right)$ is reducible. We have to adopt a weighted Sobolev norm $\|*\|_{W_{\delta}^{2, k}}$ which is in the form $\|f\|_{W_{\delta}^{2, k}}:=$ 
$\sum_{i=1}^{k}\left\{\int e^{\delta t}\left|\nabla^{k} f\right|^{2}\right\}^{\frac{1}{2}}$ in the cylindrical end and agrees with the usual one in the complement. Here $\delta$ is a sufficiently small positive constant and $f$ stands for a 1-form, a section of the spinor bundle and so on. Anyway, we can construct the moduli spaces $\mathcal{M}_{i}$ according to the standard procedure.

Any solution $\left(\Phi_{2}, B_{2}\right)$ is irreducible since $\Phi_{2}$ approaches asymptotically to the unit length section $\mathbb{I}$ at the infinity of the conical end. As for $\left(\Phi_{1}, B_{1}\right)$, its irreducibility is assured by the assumption that $b_{2}^{+}\left(X_{1}\right)>0$. In fact, we can choose $\mu_{1}$ from the complement of the affine subspace

$$
F_{B_{b}}^{+}-\tau \cdot \operatorname{Pr}^{+}\left\{\pi^{*}\left(\mu_{m}\right)\right\}+\text { Image }\left\{d^{+}: \Omega_{W_{\delta}^{k, 2}}^{1}\left(X_{1}\right) \mapsto \Omega_{W_{\delta}^{k-1,2}}^{+}\left(X_{1}\right)\right\}
$$

because the codimension is no less than 1 . Here $B_{b}$ is a fixed base point of connections such that $\left.F_{B_{b}}^{+}\right|_{M_{\Gamma} \times[1, \infty)}=\operatorname{Pr}^{+}\left\{\pi^{*}\left(\mu_{m}\right)\right\}$. The second line of the equation prevents $\Phi_{1}$ from vanishing identically.

Thus we have

Proposition 10.6. $\quad$ 1. If $\mathcal{M}_{R}$ are non-empty for all sufficiently large $R$, then neither $\mathcal{M}_{1}$ nor $\mathcal{M}_{2}$ are empty.

2. If $b_{2}^{+}\left(X_{0}\right)>0$ and if the perturbation is generic, $\mathcal{M}_{i}$ are finite dimensional smooth manifolds such that $\operatorname{dim} \mathcal{M}=\operatorname{dim} \mathcal{M}_{1}+\operatorname{dim} \mathcal{M}_{2}+1$.

Remark 10.7. The term 1 in the right-hand side of the formula in (2) is the dimension of $U(1)$, which is the isotropy subgroup at $\left(0, B_{0}\right)$ of the gauge group.

Now suppose $(s, \varrho)$ to be $\left(s_{\tilde{\omega}}\right.$, id). Then $\operatorname{dim} \mathcal{M}_{R}=0$ and $\mathcal{M}_{R}$ is non-empty (see Section 1). Then the first assertion of Proposition 10.6 implies that $\mathcal{M}_{1}$ and $\mathcal{M}_{2}$ are both non-empty. But the second assertion of Proposition 10.6 implies that either $\mathcal{M}_{1}$ or $\mathcal{M}_{2}$ is a negative dimensional manifold and thus must be empty. This is a contradiction.

\section{ApPEndix}

We will prove Lemma 6.3.

Denote by $L$ the operator $\triangle+\frac{r}{2}|\alpha|^{2}$ acting on $C_{0}^{\infty}(X)$.

Lemma 11.1. There exist a compact set $K \subset X$ and a positive constant $C$ such that, for an arbitrary $f \in C_{0}^{\infty}(X)$, it holds that

$$
\|f\|_{W_{0}^{2.2}(X)} \leq C\left(\|L f\|_{L^{2}(X)}+\|f\|_{L^{2}(K)}\right) .
$$

Proof. A short calculation shows that

$$
\nabla^{*} \nabla \nabla f=\nabla \triangle f+\operatorname{Ric}(\nabla f, *)
$$

where Ric means the Ricci curvature of the Riemannian metric. Taking the inner product of both sides with $\nabla f$, integrating the result over $X$ and using an integration by parts, we obtain

$$
\int_{X}|\nabla \nabla f|^{2}=\int_{X}|\triangle f|^{2}+\int_{X} \operatorname{Ric}(\nabla f, \nabla f) .
$$

On the other hand, the very definition of $L$ and an integration by parts imply that

$$
\int_{X}|\nabla f|^{2}=\int_{X}\langle L f, f\rangle-\int_{X} \phi|f|^{2}
$$


where $\phi$ denotes the function $\frac{r}{2}|\alpha|^{2}$. Choose a compact set $K$ sufficiently large so that $\phi \geq \frac{1}{2}$ on the complement of $K$. (See Proposition 3.2.) Then Hölder's inequality implies that

$$
\int_{X}|\nabla f|^{2} \leq \frac{1}{2} \int_{X}|L f|^{2}+\frac{1}{2} \int_{K}|f|^{2}
$$

Further, integrating the identity

$$
|\triangle f|^{2}=|L f|^{2}-2\langle\triangle f, \phi f\rangle-\phi^{2}|f|^{2}
$$

over $X$ and making use of an integration by parts and Schwarz' inequality, we obtain

$$
\int_{X}|\triangle f|^{2} \leq \int_{X}|L f|^{2}-\int_{X}\left(2 \phi|\nabla f|^{2}+\phi^{2}|f|^{2}\right)+2 \int_{X}|f| \cdot|\nabla f| \cdot|\nabla \phi| .
$$

By choosing $K$ sufficiently large, we may assume that $|\nabla \phi| \leq \frac{1}{4}$ on the complement of $K$. (See Proposition 3.2.) Thus we obtain

$$
\int_{X}|\triangle f|^{2} \leq \int_{X}|L f|^{2}-\frac{1}{8} \int_{X-K}\left(|\nabla f|^{2}+|f|^{2}\right)+C \int_{K}\left(|\nabla f|^{2}+|f|^{2}\right) .
$$

The weak convexity implies that $|\operatorname{Ric}|$ is bounded over $X$. Thus combining the inequalities (11.1), (11.3) and (11.4), we get the required inequality.

Proof of Lemma 6.3. It is trivial that $L$ extends uniquely to the symmetric operator $\tilde{L}$ over $L^{2}(X)$ with domain $W_{0}^{2,2}(X)$.

First, we will show that $\tilde{L}$ is self-adjoint. Suppose that $u, v \in L^{2}(X)$ satisfy

$$
\langle u, \tilde{L} f\rangle=\langle v, f\rangle
$$

for an arbitrary $f \in W_{0}^{2,2}(X)$. Let $\left\{u_{n}\right\}_{n \in \mathbb{N}} \subset C_{0}^{\infty}(X)$ converges strongly to $u$ with respect to the $L^{2}(X)$ norm. It follows by using an integration by parts that

$$
\left\langle u_{n}, \tilde{L} f\right\rangle=\left\langle L u_{n}, f\right\rangle
$$

which implies that $\left\{L u_{n}\right\}_{n \in \mathbb{N}}$ converges weakly to $v$ with respect to the $L^{2}(X)$ norm. Thus $\left\{\left\|L u_{n}\right\|_{L^{2}(X)}\right\}_{n \in \mathbb{N}}$ is bounded. Then Lemma 11.1 implies $\left\{\left\|u_{n}\right\|_{W^{2,2}(X)}\right\}_{n \in \mathbb{N}}$ is also bounded. By passing to a suitable subsequence, we may assume that $\left\{u_{n}\right\}_{n \in \mathbb{N}}$ converges weakly to an element $u_{0} \in W_{0}^{2,2}(X)$ with respect to the $W^{2,2}(X)$ norm. It follows from (11.5) that $u_{0}=u$ almost everywhere, that is, $u \in W_{0}^{2,2}(X)$.

Second, we will show

Lemma 11.2. If a sequnce $\left\{u_{n}\right\}_{n \in \mathbb{N}} \subset W_{0}^{2,2}(X)$ satisfies that $\lim _{n \rightarrow \infty}\left\|\tilde{L} u_{n}\right\|_{L^{2}(X)}=$ 0 and $\left\|u_{n}\right\|_{L^{2}(K)}=1$, then $\left\{u_{n}\right\}_{n \in \mathbb{N}}$ converges strongly to an element $u_{0} \in \operatorname{Ker} \tilde{L}$ with respect to the $W^{2,2}(X)$ norm.

It is easy to check that this lemma derives immediately the closedness of $\operatorname{Im} \tilde{L}$ in $L^{2}(X)$ and the finite dimensionality of $\operatorname{Ker} \tilde{L}$.

The inequality (11.0) implies that $\left\{u_{n}\right\}_{n \in \mathbb{N}}$ is bounded in $W_{0}^{2,2}(X)$. Thus by passing to a suitable subsequence, we may assume that $\left\{u_{n}\right\}_{n \in \mathbb{N}}$ converges weakly to an element $u_{0}$ with respect to the $W_{0}^{2,2}(X)$ norm. Then the Sobolev embedding theorem implies that $\left\{\left.u_{n}\right|_{K}\right\}_{n \in \mathbb{N}}$ converges strongly to $\left.u_{0}\right|_{K}$ in $L^{2}(X)$. (It is this part where we have to use the compactness of $K$.) Applying (11.0) to $\left\{u_{n}-u_{0}\right\}_{n \in \mathbb{N}}$, we can show that $\left\{u_{n}\right\}_{n \in \mathbb{N}}$ converge strongly to $u_{0}$ in $W_{0}^{2,2}$. 
Third, we will show that $\operatorname{Ker} \tilde{L}=\{0\}$, which in the same time implies that Coker $\tilde{L}=\{0\}$. Suppose $\tilde{L} u=0$. The local elliptic regularity means that $u$ is smooth. On the other hand, (11.2) implies that $\int_{X}|\nabla u|^{2}=0$. Thus $u$ is a constant function. Since $u \in L^{2}(X), u \equiv 0$.

Remark 11.3. Due to the weak convexity, we can show that $W_{0}^{2,2}(X)$ coincides with the function space $W^{2,2}(X)$ that consists of $L^{2}$-functions whose distributional derivatives of order 1 and 2 are realized as $L^{2}$-functions. (See [K-M2].)

\section{REFERENCES}

[E] Y.Eliashberg, Contact 3-manifolds, twenty years since Martinet's work, Ann. Inst. Fourier. 42 (1992), 165-192. MR 93k:57029

[F] K.Fløyshov, The Seiberg-Witten equations and 4-manifolds with boundary, Math. Res. Lett. 3 (1996), 373-390. MR 97i:57037

[Gr] M.Gromov, Pseudo-holomorphic curves in symplectic manifolds, Invent. Math. 82 (1985), 307-347. MR 87j:53053

[G-T] D.Gilbarg and N.S.Trudinger, Elliptic partial differential equalities of second order, 2nd ed., Springer-Verlag, 1983. MR 86c:35035

[K-M1] P.B.Kronheimer and T.S.Mrowka, The genus of embedded surfaces in the projective plane, Math. Res. Lett. 1 (1994), 797-808. MR 96a:57073

[K-M2] , Monopoles and contact structures, Inv. Math. 130 (1997), 209-255. MR 98h:57058

[Ko] D.Kotschick, The Seiberg-Witten invariants of symplectic four-manifolds [after C.H.Taubes], Séminaire Bourbaki 48ène année nº 812 (1995-1996). MR 98h:57057

[Ma] R.Mandelbaum, Irrational connected sums, Trans. Amer. Math. Soc. 247 (1979), 137156. MR 80e:57023

[M-S-T] J.W.Morgan, Z.Szabó and C.H.Taubes, A product formula for the Seiberg-Witten invariants and the generalized Thom conjecture, Jour. Diff. Geom. 44 (1996), 706-788. MR 97m:57052

[O-O] H.Ohta and K.Ono, Simple singularities and topology of symplectically filling 4-manifold, Comment. Math. Helv. 74 (1999), 575-590. CMP 2000:06

[T1] C.H.Taubes, $S W \Rightarrow G r$ : From the Seiberg-Witten equations to pseudo-holomorphic curves, J. Amer. Math. Soc. 9 (1996), 845-918. MR 97a:57053

[T2] $\longrightarrow G r \Rightarrow S W$ : From pseudo-holomorphic curves to Seiberg-Witten solutions, J. Differential Geom. 51 (1999), 203-334. MR 20001:53123

[T3] Counting pseudo-holomorphic curves in dimension 4, preprint.

[W] E.Wittem, Monopoles and four-manifolds, Math. Res. Lett. 1 (1994), 769-796. MR 96d:57035

Department of Mathematics, Hokkaido University, Sapporo 060-0810, Japan

E-mail address: kanda@math.sci.hokudai.ac.jp 